TecnoLógicas

ISSN-p 0123-7799

ISSN-e 2256-5337

Vol. 22, No. 46, pp 39-60

Sep-dic de 2019

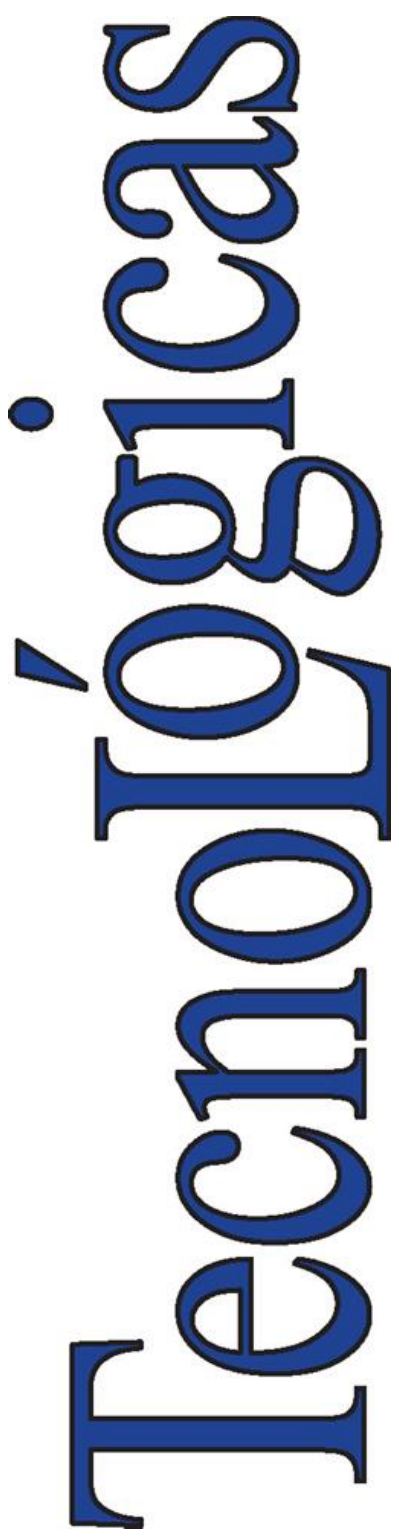

(C) Instituto Tecnológico Metropolitano Este trabajo está licenciado bajo una Licencia Internacional Creative Commons Atribución (CC BY-NC-SA)

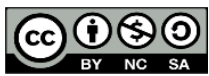

Artículo de Investigación/Research Article

\section{Métodos estadísticos para la evaluación de la susceptibilidad por movimientos en masa}

\section{The Susceptibility of Landslide Evaluation and Zoning by Statistical Methods}

\author{
Edier Aristizábal(D), Mariana Vásquez (D) 2 \\ y Diana Ruíz (iD)
}

Recibido: 05 de marzo de 2019

Aceptado: 19 de junio de 2019

Cómo citar / How to cite

E. Aristizabal, M. Vásquez y D. Ruiz, "Métodos estadísticos para la evaluación de la susceptibilidad por movimientos en masa". TecnoLógicas, vol. 22, no. 46, pp. 39-60, 2019.

https://doi.org/10.22430/22565337.1247

1 Ingeniero geólogo, Departamento de Geociencias y Medio Ambiente, Universidad Nacional de Colombia, Medellín-Colombia,

evaristizabalg@unal.edu.co

2 Ingeniera geóloga, Departamento de Geociencias y Medio Ambiente, Universidad Nacional de Colombia, Medellín-Colombia, mvasquez@unal.edu.co

3 Ingeniera geóloga, Universidad EAFIT, Medellín-Colombia, diruva123@gmail.com 


\section{Resumen}

Existen diferentes métodos que permiten establecer a escalas regionales la susceptibilidad a la ocurrencia de movimientos en masa. Entre los métodos más utilizados se encuentran los métodos estadísticos bivariado y multivariado, los cuales exigen un inventario de procesos de remoción en masa. En el presente estudio se evalúa y zonifica la susceptibilidad por movimientos en masa en el norte de los Andes colombianos, región conocida como valle de Aburrá, por dos métodos estadísticos, uno de ellos bivariado, denominado Peso de la Evidencia, y recomendado por el Servicio Geológico Colombiano para estudios de amenaza en suelos rurales; y un segundo método estadístico tipo multivariado, denominado Regresión Logística, de amplio uso a nivel mundial. Para ambos casos, la construcción del modelo de susceptibilidad se realizó soportado en el histograma de frecuencias, correlación de Pearson, Análisis Discriminante y Análisis de Componentes Principales. Para evaluar el desempeño, la capacidad de predicción y los criterios de zonificación en alto, medio y bajo de cada uno de los métodos utilizados se utilizó el análisis ROC. Para la regresión logística se obtuvo un área bajo la curva del $76.8 \%$ para el desempeño y $77.5 \%$ para la capacidad de predicción, mientras que para el Peso de la Evidencia se obtuvo un $77.8 \%$ en el desempeño y $77.5 \%$ en la predicción, señalando resultados satisfactorios que permiten la incorporación de dichos resultados en los estudios básicos necesarios para la ordenación del territorio.

\section{Palabras clave}

Peso de la evidencia, regresión logística, amenaza, movimientos en masa, Valle de Aburrá.

\section{Abstract}

Evaluating and zoning mass movement landslide is a fundamental tool for land planning. There are different methods that help to establish in a regional scale landslide hazard. The most common methods are bivariate statistics and multivariate statistic, which need a landslide historical inventory. This study takes a place in the North of the Andes in a region of Colombia called Aburrá Valley, for evaluating and zoning landslide susceptibility by two methods, one of them is bivariate statistics called Weights of Evidence which is recommended by the Geological Service of Colombia for rural area, and the second one is a multivariate statistic method, called logistic regression, which is widely used worldwide. Both methods are supported in frequency histogram, Pearson correlation, Discriminant Analysis, and Principal Component Analysis. The accuracy of the landslide susceptibility maps produced from the two models is classified in high, medium and low by ROC analysis. The AUC plot estimation results showed that the susceptibility map using Logistic regression has a training accuracy of $76.5 \%$ and a prediction capacity of $77.5 \%$. The Weights of evidence method has the highest training accuracy of $77.8 \%$ and a prediction of $77.5 \%$. This result allows to include the methods in territorial planning studies.

\section{Keywords}

Weight of Evidence, logistic regression, hazard, mass movements, Valle de Aburrá. 


\section{INTRODUCCIÓN}

La evaluación y zonificación de la susceptibilidad por movimientos en masa es una herramienta fundamental para la ordenación del territorio en ambientes montañosos y condiciones tropicales. La susceptibilidad por movimientos en masa es definida como la probabilidad de ocurrencia en un área específica, de acuerdo con las condiciones intrínsecas del terreno, donde factores detonantes, tales como precipitación o sismos no son considerados en su análisis [1], [2]. La susceptibilidad es el primer acercamiento hacia la evaluación de la amenaza, debido a que debe explicar la distribución espacial de los movimientos en masa. Por lo tanto, los mapas de susceptibilidad zonifican el terreno de acuerdo con la favorabilidad a fallar de una ladera, con el propósito de analizar posteriormente los mecanismos detonantes.

Los métodos para la evaluación y la zonificación de la susceptibilidad por movimientos en masa pueden ser divididas en métodos cualitativos y métodos cuantitativos [3], [4]. Los métodos cualitativos, denominados métodos basados en el conocimiento o métodos heurísticos, se fundamentan en el mapeo geomorfológico directo o mapeo indirecto, a través de pesos que son otorgados a las diferentes variables y/o clases de acuerdo con el criterio de experto [5]. Aunque estos métodos permiten incorporar en el análisis una gran cantidad de variables complejas, dependen completamente de la experiencia y conocimiento del experto sobre la ocurrencia de movimientos en masa en la región específica de estudio y los factores de causa, por lo que son considerados con un alto grado de subjetividad [6].

A su vez, los métodos cuantitativos utilizan expresiones matemáticas objetivas produciendo factores de seguridad, índices de confiabilidad o probabilidades de ocurrencia de movimientos en masa [3], [6], [7]. Entre estos, los métodos denominados con base física utilizan modelos geotécnicos, y en algunas ocasiones acoplados con modelos hidrológicos, para estimar el factor de seguridad de cada unidad de análisis [8][10]. A su vez, los métodos estadísticos establecen relaciones funcionales entre factores de inestabilidad y la distribución pasada y presente de los movimientos en masa. Estas relaciones generalmente corresponden a funciones de densidad de movimientos en masa que pueden ser establecidas para cada factor individual, denominados métodos estadísticos bivariados, o relaciones combinadas entre una variable dependiente (ocurrencia de movimientos en masa) y variables independientes (factores de causa) denominados métodos estadísticos multivariados [6]. Aunque estos métodos han sido de amplia aplicación a escalas regionales, exigen tener un exhaustivo inventario de movimientos en masa. Entre los métodos estadísticos bivariados se destacan: Radio de Frecuencia [11], Índice Estadístico [12], Peso de la Evidencia [13], y Funciones de Favorabilidad [14]. Entre los métodos multivariados de mayor uso están: Análisis Discriminante [15], Regresión Logística [16], y Análisis Condicional [17], [18].

En el presente trabajo, se implementaron los dos métodos estadísticos de mayor aplicación [16], [19], [20] para la evaluación y zonificación de la susceptibilidad por movimientos en masa en el valle de Aburrá. El método estadístico bivariado, denominado Peso de la Evidencia (WoE, por sus siglas en inglés) y recomendado para su aplicación por el Servicio Geológico Colombiano; y el método estadístico multivariado, denominado Regresión Logística (RL). Ambos modelos se implementaron utilizando las mismas variables. El desempeño de ambos modelos y su capacidad de predicción fue evaluado cuantitativamente a través de la Curva de Éxito, Curva de Predicción. Finalmente, se seleccionó el modelo con el mejor 
desempeño y capacidad de predicción, y se clasificó en categorías de acuerdo con la Matriz de Confusión y Curva ROC [21].

\section{2. ÁREA DE ESTUDIO}

El valle de Aburrá está localizado en el norte de la cordillera Central de los Andes colombianos, con una longitud aproximada de $80 \mathrm{~km}$ y una amplitud variable de 30 $\mathrm{km}$ en el sector más ancho y $10 \mathrm{~km}$ en el sector más angosto. Comprende un área de $1,152 \mathrm{~km}^{2}$. El valle de Aburrá está conformado por 10 municipios, de sur a norte son: Caldas, La Estrella, Sabaneta, Itagüí, Envigado, Medellín, Bello, Copacabana, Girardota y Barbosa. El presente estudio se realizó para siete de dichos municipios, exceptuando Sabaneta, Medellín y Girardota, los cuales no fueron incluidos en el área de estudio, debido a que ya contaban con mapas de susceptibilidad por movimientos en masa de su territorio (Fig. 1).
Colombia, y el valle de Aburrá, ha sido un territorio fuertemente afectado por fenómenos de origen natural, especialmente por movimientos en masa y flujos torrenciales [22]-[25], donde se destacan los trágicos eventos de Media Luna en 1954 con aproximadamente 100 muertos, Santo Domingo en 1974 con 70 muertos, Villatina en 1987 con más de 500 personas reportadas como víctimas fatales, y recientemente La Gabriela en el año 2010 , con 85 muertos.

Geomorfológicamente, el Valle de Aburrá se describe como un cañón entallado entre un sistema de altiplanos, y dividido en tres segmentos de acuerdo con las características generales de forma, ancho, orientación y contexto geomorfológico. Un primer segmento, denominado valle de Aburrá superior, caracterizado por ser un valle estrecho con forma de "V" asimétrica, con una marcada influencia estructural sobre ladera occidental asociada al sistema de fallas de San Jerónimo [26].

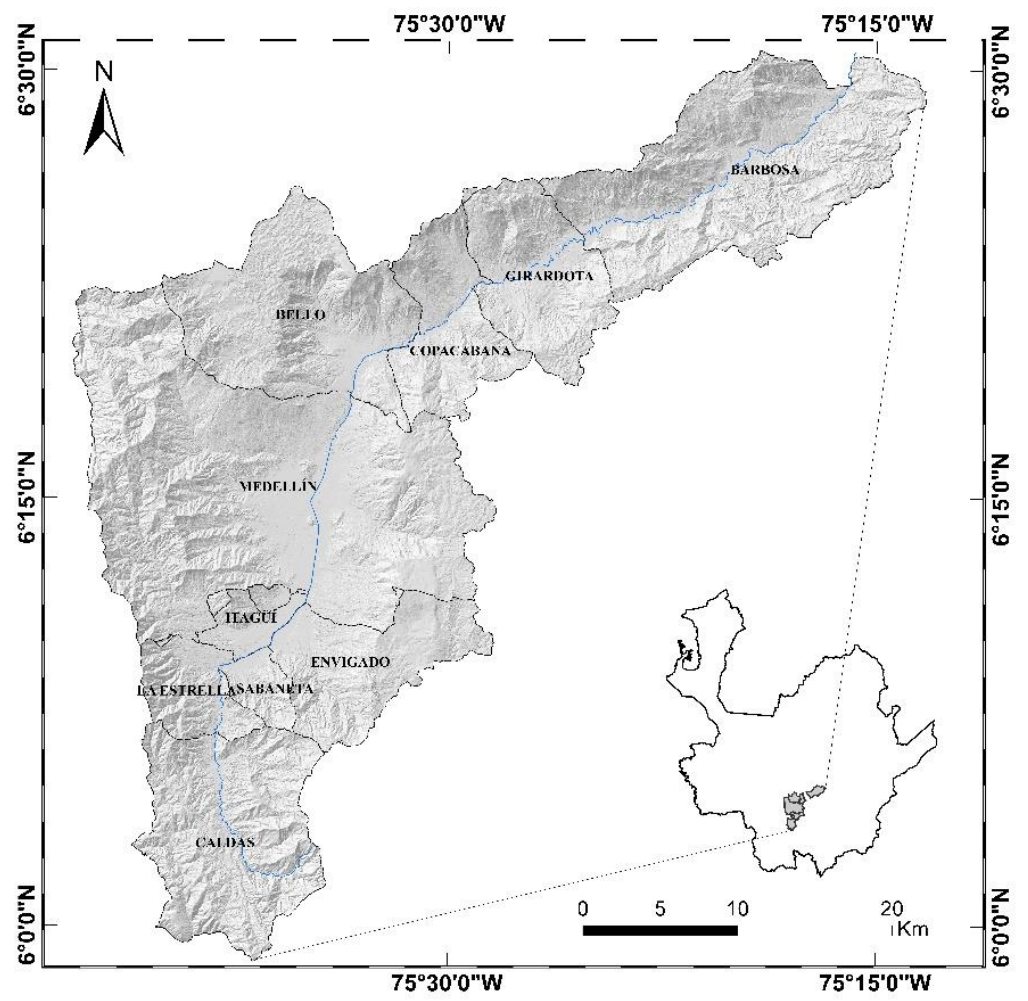

Fig. 1. Localización del área de estudio. Fuente: autores. 
El valle de Aburrá medio es un valle más ancho, con fondo plano y amplio; sobre las vertientes se presentan superficies suaves en depósitos de vertiente, sistemas de colinas y lomos. El valle de Aburrá inferior, se caracteriza por ser un valle más estrecho, simétrico y de flancos paralelos, en el fondo se desarrolla una llanura aluvial de amplitud moderada y sobre las vertientes se identifica gran cantidad de depósitos aluviales, con evidencias de rasgos morfotectónicos en ambas vertientes [27].

\section{DATOS Y METODOLOGÍA}

Para la evaluación y zonificación de la susceptibilidad por movimientos en masa, [28] recomienda la ejecución de cinco pasos: (i) la identificación y mapeo de los movimientos en masa en el área de estudio; (ii) la identificación y mapeo de los factores condicionantes que están directa o indirectamente correlacionados con la ocurrencia; (iii) la estimación de la contribución relativa de cada factor condicionante en la ocurrencia de los movimientos en masa; (iv) la zonificación del terreno en dominios con diferentes niveles de susceptibilidad; y (v), la evaluación del desempeño del modelo y su capacidad de predicción.

A continuación, se describen metodológicamente cada uno de estos pasos implementados en el presente estudio. En el paso dos se incluye como actividad adicional la evaluación y selección objetiva de los factores condicionantes, que permite elaborar el modelo de susceptibilidad de mayor eficacia y eficiencia en la identificación de áreas susceptibles a la ocurrencia de movimientos en masa. Los pasos cuatro y cinco se agrupan en un mismo ítem, ya que se realizan con las mismas herramientas.

\subsection{Inventario de movimientos en masa}

El inventario de movimientos en masa es una herramienta necesaria para la evaluación de la susceptibilidad a través de métodos estadísticos. Además, constituyen la principal fuente de información con fines de calibración y validación de los resultados de los mapas de zonificación de la susceptibilidad [29], [30].

El inventario para el valle de Aburrá fue ejecutado mediante un levantamiento histórico de eventos y fotointerpretación multitemporal, con un posterior trabajo de campo para verificar y ajustar el inventario. La base de datos histórica de movimientos en masa se basó en el Sistema de Inventarios de Desastres (DesInventar) [31], y el Sistema de Información de Movimientos en Masa SIMMA-[32]. El DesInventar (http://www.desinventar.org/) es un software que permite la sistematización, de los desastres históricos y el análisis de estos desde un punto de vista espacial y temporal. A su vez el SIMMA (http://simma.sgc.gov.co/), desarrollado e implementado por el Servicio Geológico Colombiano (SGC), dispone de registros de movimientos en masa desde el año 1917.

En cuanto al proceso de fotointerpretación, se enfocó en identificar los procesos morfodinámicos presentes en el área de estudio activos o inactivos, y estos últimos en latentes o recientes $\mathrm{y}$ relictos, de acuerdo con las definiciones de Cruden \& Varnes [33]. Para lo cual se utilizaron fotografías aéreas a color con escalas 1:10.000 y 1:5.000 tomadas entre los años 2011 y 2014 por el Instituto Geográfico Agustín Codazzi (IGAC), que cubren la totalidad del valle de Aburrá.

$\mathrm{El}$ inventario de movimientos en masa fue verificado en campo, donde se ajustaron la localización y extensión de los eventos históricos y fotointerpretados, y se adicionaron eventos recientes que no aparecían registrados. 


\subsection{Selección de variables condicionantes}

Las variables condicionantes, también denominadas variables ambientales, intrínsecas o de predisposición, se definen como las características internas e inherentes del terreno que favorecen la ocurrencia de movimientos en masa [34]. Dichas variables presentan poca variación temporal, por lo que son denominadas por algunos autores como variables estáticas o cuasi-estáticas. En general, se pueden dividir en variables continuas, que pueden expresarse numéricamente y son susceptibles de medirse; y variables discretas o categóricas, que expresan una cualidad, característica o atributo, que solo permite clasificar en rangos o categorías. Las variables continuas, en general, corresponden a variables morfométricas obtenidas a partir del DEM. Entre tanto, las variables categóricas se pueden clasificar en variables dicotómicas, las cuales pueden tomar solo dos rangos; y variables politómicas, que contienen diferentes rangos.

En la literatura existe una gran cantidad de posibles variables condicionantes, lo que convierte en un proceso más complejo la evaluación de la susceptibilidad. Este conjunto de variables que influyen directa o indirectamente en la ocurrencia de movimientos en masa difiere dependiendo de la escala de análisis, características de la región de estudio y el tipo de movimiento en masa [35]. Cada región presenta un conjunto de variables propias que responden a la ocurrencia de los movimientos en masa. En este sentido, en el presente estudio se utilizan, en una primera fase, un rango amplio de variables utilizadas a nivel mundial o recomendadas en estudios locales, como la guía del SGC (2015).

Las variables continuas inicialmente preseleccionadas en el modelo de susceptibilidad son: pendientes, aspecto, rugosidad, curvatura, índice topográfico de humedad, índice de relieve relativo, inclinación de la ladera, índice de posición topográfica, distancia relativa al cauce, $\mathrm{y}$ distancia a fallas. Dichas variables fueron generadas a partir de un modelo de elevación del terreno, con resolución espacial de $5 \mathrm{~m}$ elaborado por el IGAC. La distancia a fallas se elaboró basados en las estructuras regionales cartografiadas [26], y para cada estructura se definieron rangos de influencia cada $100 \mathrm{~m}$ hasta los $1500 \mathrm{~m}$, el cual, de acuerdo con evidencia de campo, se consideró el área de afectación del macizo rocoso. Entre las variables categóricas politómicas se contó con la geología y coberturas del suelo.

Las variables: pendientes, aspecto, rugosidad, curvatura, índice de relieve relativo, e índice de posición topográfica, se calcularon con el software ArcMap 10.4.1 y el conjunto de herramientas de superficie Dem Surface Tools, Terrain Tools, Focal Statistics, y Land Facet Corridor Tools. Las variables: inclinación de la ladera, índice de topográfico de humedad (TWI), y distancia relativa al cauce, se calcularon con el software Whitebox GAT 3.4 y la herramienta Downslope Index, Wetness Index y Downslope Distance to Stream, respectivamente.

Las variables geología y distancia a fallas, se tomaron del estudio del Area metropolitana del Valle de Aburra -AMVA (2007). Para el mapa de coberturas del suelo, se realizó una clasificación supervisada con el software Envi 5.3 con la herramienta Likehood clasification, a partir de ortofotos con resolución espacial de $0,15 \mathrm{~m}$ para suelos urbanos y de $0,3 \mathrm{~m}$ para la zona rural de los municipios.

Para la selección del grupo de variables mejor predictoras para las condiciones locales del valle de Aburrá, se trabajó con cuatro métodos: (i) análisis de frecuencia de variables [36,] el cual es un método de distribución espacial que evidencia el comportamiento de cada variable en comparación con la presencia o ausencia de movimientos en masa; (ii) la correlación de Spearman, que permite evaluar la relación 
entre dos variables continuas y descartar variables que generan ruido en la aplicación de los modelos de susceptibilidad; (iii) Análisis Discriminante, técnica multivariada que permite encontrar diferencias entre grupos de variables, en matrices de correlación, análisis ANOVA y pruebas de M de box; y finalmente, (iv) análisis de Componentes Principales, el cual consiste en encontrar transformaciones ortogonales de las variables originales para conseguir un nuevo conjunto de variables, que permite identificar las variables que aportan mayor cantidad de información independiente al modelo de susceptibilidad. Las variables con mayor capacidad de discriminar entre la ocurrencia y no ocurrencia de movimientos en masa fueron entonces seleccionadas para su incorporación dentro del modelo estadístico de susceptibilidad por movimientos en masa para el valle de Aburrá.

\subsection{Modelos de susceptibilidad}

En el presente estudio se implementaron dos métodos de evaluación y zonificación de la susceptibilidad aplicable para movimientos en masa. El método estadístico bivariado denominado Peso de la Evidencia (WoE) [13], [37], y el método estadístico multivariado denominado Regresión Logística (RL) [15], [38].

El método estadístico bivariado denominado WoE está basado en la teoría de la probabilidad bayesiana, donde se analiza la relación entre dos sucesos [39]. Para el caso de los movimientos en masa, se evalúa la relación de las áreas afectadas por los movimientos en masa y la distribución espacial de los factores condicionantes del terreno [40], [41]. Este método está fundamentalmente basado en calcular los pesos de las clases que conforman las variables condicionantes [42]. Dicho peso indica la presencia e influencia de la clase como parámetro en la ocurrencia del movimiento en masa [41], [43].

Los pesos asignados a cada clase pueden ser pesos positivos (W+) y negativos (W-). Los pesos positivos (W+) indican la presencia de la clase como parámetro que favorece los movimientos en masa y su magnitud indica su correlación. El peso negativo (W-) indica la ausencia de la clase. En caso de ser cero, indica que la clase analizada no es de interés para el fenómeno por movimiento en masa [41], [44]. Los pesos son definidos en (1):

$$
\mathrm{W}^{+}=\operatorname{Ln} \frac{\frac{\mathrm{A} 1}{\mathrm{~A} 1+\mathrm{A} 2}}{\frac{\mathrm{A} 3}{\mathrm{~A} 3+\mathrm{A} 4}} \mathrm{~W}^{-}=\operatorname{Ln} \frac{\frac{\mathrm{A} 2}{\mathrm{~A} 1+\mathrm{A} 2}}{\frac{\mathrm{A} 4}{\mathrm{~A} 3+\mathrm{A} 4}}
$$

Donde A1 es el número de pixeles con movimientos en masa en la clase; A2 número de pixeles con movimientos en masa que no están presentes en la misma clase; A3 número de pixeles en la clase que no hay movimientos en masa; y A4 es el número de pixeles en la clase donde no hay movimientos en masa y que no está presente en la misma clase [45].

Para cada variable, entonces, se estima el Peso de Contraste (C) dado en (2):

$C=w^{+}-w^{-}$

Donde $\mathrm{C}$ refleja el espacio de asociación respecto a la clase en la predicción del movimiento en masa, la cual es cero cuando la clase no afecta; es positivo, cuando existe una asociación positiva; y negativo, cuando existe una asociación negativa a la distribución de los movimientos en masa [46].

Para los pesos de cada clase (Xn), se utilizó el valor del peso de contraste (C) recomendado por el SGC (2017). El Índice de Susceptibilidad (IS) para cada celda que compone el área del proyecto se estima utilizando (3):

$$
I S=C_{1} X_{1}+C_{2} X_{2}+C_{3} X_{3}+\cdots+C_{n} X_{n}
$$


El método estadístico multivariado, denominado RL, estima la relación entre una variable dependiente (la ocurrencia de movimientos en masa) de valores dicotómicos de 0 (no ocurrencia) y 1 (si ocurrencia), con un grupo de variables independientes, en este caso las variables condicionantes del terreno. La ventaja de la $\mathrm{RL}$ es que dichas variables predictoras no requieren tener distribución normal y pueden ser discretas o continuas (categóricas o no categóricas), o una combinación de ambas [47]. La relación de dependencia entre la ocurrencia de movimientos en masa y las variables es expresada cuantitativamente como en (4):

$$
\mathrm{P}(\mathrm{y})=1 /\left(1+\mathrm{e}^{-\mathrm{z}}\right)
$$

Donde P(y) es la probabilidad especial estimada de la ocurrencia de movimientos en masa y se encuentra en el rango de 0 a 1. y z es la siguiente combinación lineal de las variables independientes (5):

$$
z=b_{0}+b_{1} x_{1}+b_{2} x_{2}+b_{3} x_{3}+b_{n} x_{n}
$$

Donde $b_{0}$ es el intercepto del modelo según el modelo de RL, los valores $b_{i}(\mathrm{i}=1$, $2,3, \mathrm{n})$ son los coeficientes de la regresión logística, es decir, los pesos de las variables, y los valores $x_{i}(\mathrm{i}=1,2,3, \ldots, \mathrm{n})$ son las variables independientes. El modelo final es una RL de la ocurrencia de movimientos en masa (presencia o ausencia) basado en las variables independientes. Para la implementación del método de RL, se utilizó el software SPSS del IBM, al cual se ingresaron $\quad 256.970 \quad$ valores que corresponden al $80 \%$ de los movimientos en masa del mapa de inventario compilado, y se seleccionaron aleatoriamente igual cantidad de pixeles que no presentaran movimientos en masa. Con el fin de evaluar la capacidad de predicción del modelo se reservó el 20 \% del inventario.

\subsection{Zonificación y evaluación de los modelos de susceptibilidad}

Existen múltiples fuentes de incertidumbre en la evaluación de la susceptibilidad por movimientos en masa, relacionadas con la evaluación de la probabilidad espacial y el análisis temporal de la probabilidad [5]. Es por esta razón que es fundamental para la aceptación o rechazo de un modelo, evaluar el desempeño y predicción. La Australian Geomechanics Society -AGC-[48] define el desempeño como la capacidad conceptual y matemática del modelo en describir el comportamiento del sistema y la sensibilidad del modelo a pequeños cambios en los parámetros de entrada, y la predicción como la exactitud en predecir los datos observados. En general, se obtiene un mayor ajuste del modelo a la evaluación por desempeño que a la capacidad de predicción, sin embargo, es más importante la capacidad de predicción [49].

En la literatura existen diferentes métodos para evaluar el desempeño $\mathrm{y}$ capacidad de predicción de los mapas de susceptibilidad obtenidos mediante los modelos implementados. Para el presente estudio se implementaron: Curva de Éxito y Curva de Predicción [49], [50]. Área Bajo la Curva (AUC) [51], y Matriz de confusión [52].

En términos de zonificación, específicamente los métodos denominados Curva de Éxito y Predicción brindan un criterio cuantitativo en términos de probabilidad espacial, que permite proponer valores de susceptibilidad, necesarios para convertir un mapa de celdas con valores continuos, en un mapa tipo semáforo con las categorías de susceptibilidad amenaza alta, media y baja. En cuanto al Área Bajo la Curva (AUC), no solo permiten evaluar el ajuste y predicción del modelo, sino que además se convierten en un criterio para seleccionar el modelo más adecuado. 


\section{RESULTADOS}

\subsection{Inventario de movimientos en masa}

Como resultado, se obtuvo que el $50 \%$ del total de registros por movimientos en masa se encontraron solo en la base de datos del SIMMA, el $39 \%$ de los registros solo se encontraron en el DesInventar y el $11 \%$ restante son registros que se encontraban en ambas bases de datos. En el proceso de unificación de las bases de datos del SIMMA y DesInventar, para el área de estudio, se encontraron 107 registros repetidos, los cuales fueron debidamente revisados y depurados. Con respecto a la localización de los registros, solo el $37 \%$ del total compilados presentan coordenadas asociadas a un punto donde sucedió el evento. Para los demás registros es posible asignar una georreferenciación a cada evento con un grado de incertidumbre, dependiendo de lo detallada o amplia de la descripción geográfica de cada registro. En este sentido, el $24 \%$ del total de registros a los cuales se les asignó una georreferenciación presentan incertidumbre baja, mientras que el $76 \%$ restante presentan incertidumbres medias y altas. En cuanto a la ubicación temporal, todos los registros presentan día, mes y año y muy pocos casos describen la hora del evento.

Se complementa el inventario con un proceso de fotointerpretación, con fotografías aéreas con resolución $0.15 \mathrm{~m}$ y $0.3 \mathrm{~m}$ trabajando escalas 1:10000 para zona rural, y 1:5000 para zona urbana, tomadas del proyecto CARTOANTIOQUIA; además de fotos históricas de las décadas de 1940 y 1980, las cuales presentan escala regional. Esta información, junto a los inventarios históricos, comprende un total de 20,437 reportes, entre los cuales se tiene carcavas y surcos son el $2.6 \%$, en coronas el $97.4 \%$ restante, el cual se divide en $87.3 \%$ para coronas activas, el $9.3 \%$ coronas relicto y el $0.8 \%$ a coronas inactivas, utilizando los criterios establecidos por [53].

La Fig. 2 presenta el inventario de movimientos en masa con los registros históricos ubicados, y las cicatrices de movimientos en masa fotointerpretadas.

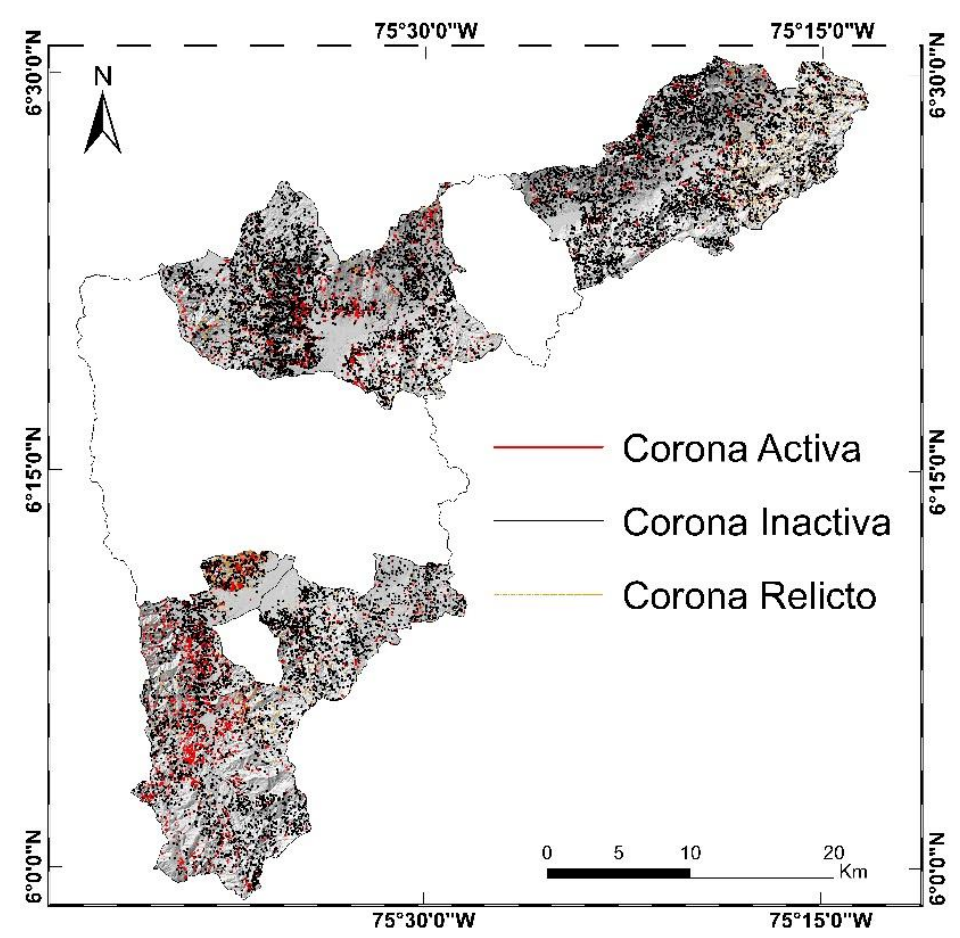

Fig. 2. Inventario de movimientos en masa. Fuente: autores. 


\subsection{Análisis exploratorio y selección de variables condicionantes}

La Fig. 3 presenta los histogramas de frecuencia para las celdas con movimientos en masa y para las celdas sin movimientos en masa. En general, se observan diferencias relevantes entre ambos histogramas señalando la buena capacidad de separación que aportan dichas variables al modelo. La única variable que no presenta un claro cambio de frecuencia entre ocurrencia y no ocurrencia de eventos es la variable curvatura tangente.
La presentan los resultados obtenidos para la correlación de Pearson de las 14 variables, resaltando las mayores correlaciones. La Tabla 1 evidencia que las variables inclinación de la ladera y pendiente son similares, y por ello no se deben incluir ambas en el modelo de susceptibilidad. Además, se resalta que el resto de las variables presentan correlaciones inferiores a 0,2 a excepción de los mapas de curvatura con valores de 0,5 , que son altos pero considerados aceptables para el modelo.
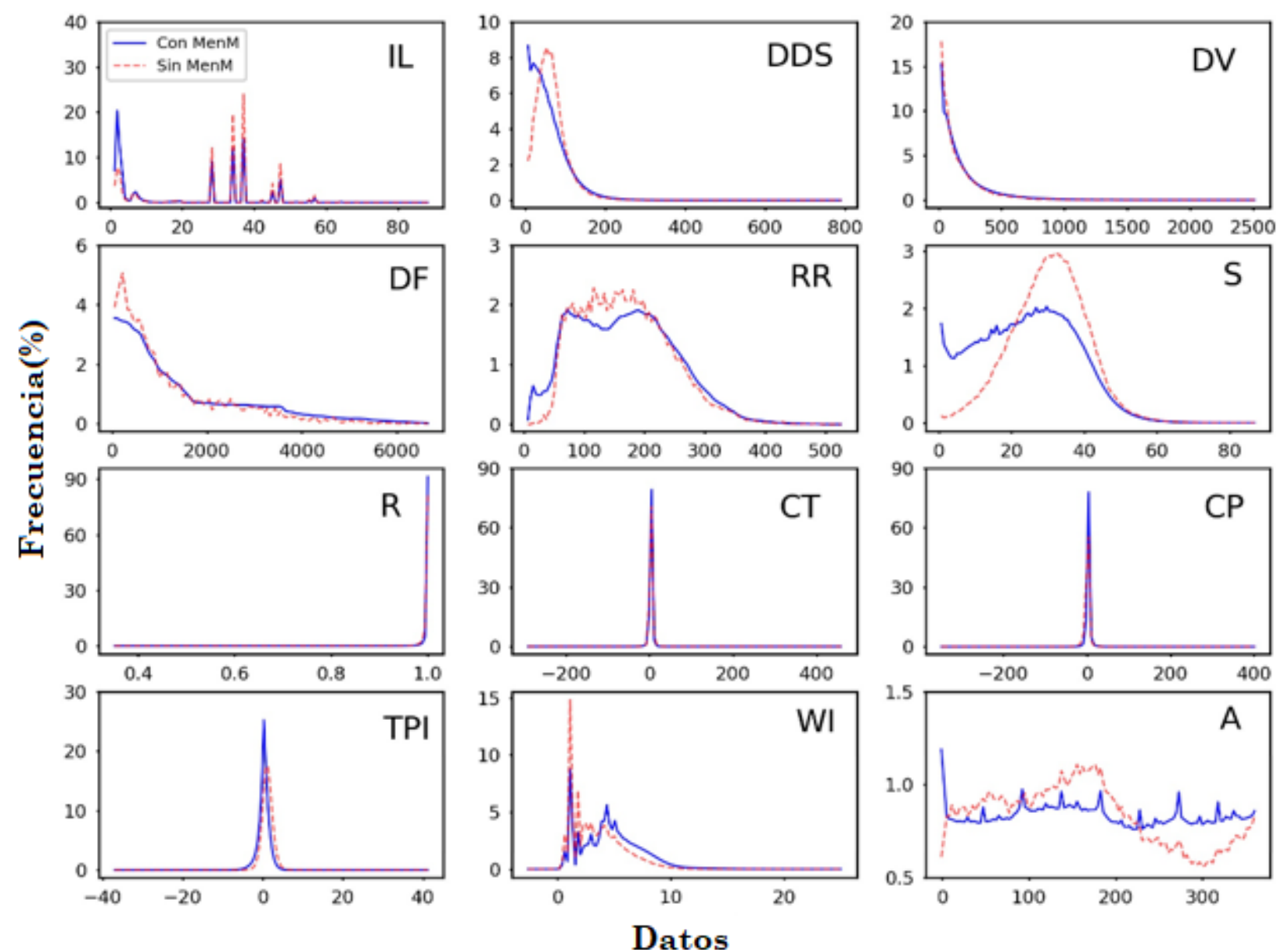

Fig. 3. Histogramas de frecuencia de cada variable con la presencia o ausencia de movimiento en masa. IL: inclinación de la ladera; DDS: distancia al drenaje siguiendo la pendiente; DV: distancia a vías; DF: Distancia a fallas RR: relieve relativo; S: pendientes; R: rugosidad; CT: curvatura tangente; CP: curvatura de perfil; TPI: Índice de posición topográfica; WI: Índice de humedad y A: aspecto. Fuente: autores. 
Métodos estadísticos para la evaluación de la susceptibilidad por movimientos en masa

Tabla 1. Valores de cada variable para la correlación de Pearson. Fuente: autores.

\begin{tabular}{|c|c|c|c|c|c|c|c|c|c|c|c|c|c|c|}
\hline & A & CP & CT & DDS & DF & DV & IL & $\mathrm{S}$ & RR & $\mathbf{R}$ & TPI & WI & COB & GEO \\
\hline A & 1,00 & 0,01 & 0,01 & 0,01 & 0,02 & 0,04 & 0,00 & 0,01 & 0,03 & 0,01 & 0,02 & 0,01 & 0,03 & 0,01 \\
\hline$\overline{C P}$ & 0,01 & 1,00 & 0,16 & 0,05 & 0,01 & 0,04 & 0,20 & 0,05 & 0,03 & 0,11 & 0,44 & 0,21 & 0,02 & 0,06 \\
\hline CT & 0,01 & 0,16 & 1,00 & 0,16 & 0,02 & 0,01 & 0,03 & 0,02 & 0,04 & 0,02 & 0,50 & 0,25 & 0,04 & 0,02 \\
\hline DDS & 0,01 & 0,05 & 0,16 & 1,00 & 0,03 & 0,05 & 0,02 & 0,01 & 0,14 & 0,08 & 0,29 & 0,15 & 0,04 & 0,00 \\
\hline DF & 0,02 & 0,01 & 0,02 & 0,03 & 1,00 & 0,05 & 0,11 & 0,11 & 0,10 & 0,03 & 0,05 & 0,05 & 0,02 & 0,18 \\
\hline DV & 0,04 & 0,04 & 0,01 & 0,05 & 0,05 & 1,00 & 0,16 & 0,17 & 0,29 & 0,03 & 0,04 & 0,06 & 0,12 & 0,11 \\
\hline IL & 0,00 & 0,20 & 0,03 & 0,02 & 0,11 & 0,16 & 1,00 & 0,90 & 0,46 & 0,12 & 0,16 & 0,48 & 0,12 & 0,05 \\
\hline $\mathbf{S}$ & 0,01 & 0,05 & 0,02 & 0,01 & 0,11 & 0,17 & 0,90 & 1,00 & 0,55 & 0,08 & 0,06 & 0,46 & 0,18 & 0,05 \\
\hline $\mathbf{R R}$ & 0,03 & 0,03 & 0,04 & 0,14 & 0,10 & 0,29 & 0,46 & 0,55 & 1,00 & 0,02 & 0,03 & 0,22 & 0,11 & 0,04 \\
\hline $\mathbf{R}$ & 0,01 & 0,11 & 0,02 & 0,08 & 0,03 & 0,03 & 0,12 & 0,08 & 0,02 & 1,00 & 0,05 & 0,03 & 0,00 & 0,01 \\
\hline TPI & 0,02 & 0,44 & 0,50 & 0,29 & 0,05 & 0,04 & 0,16 & 0,06 & 0,03 & 0,05 & 1,00 & 0,37 & 0,04 & 0,04 \\
\hline WI & 0,01 & 0,21 & 0,25 & 0,15 & 0,05 & 0,06 & 0,48 & 0,46 & 0,22 & 0,03 & 0,37 & 1,00 & 0,06 & 0,00 \\
\hline COB & 0,03 & 0,02 & 0,04 & 0,04 & 0,02 & 0,12 & 0,12 & 0,18 & 0,11 & 0,00 & 0,04 & 0,06 & 1,00 & 0,09 \\
\hline GEO & 0,01 & 0,06 & 0,02 & 0,00 & 0,18 & 0,11 & 0,05 & 0,05 & 0,04 & 0,01 & 0,04 & 0,00 & 0,09 & 1,00 \\
\hline
\end{tabular}

La Tabla 2 presenta los resultados del análisis discriminante utilizando la matriz de correlación de grupos combinados y tabla ANOVA, a través del programa SPSS. El análisis discriminante permite evaluar la diferencia entra varios grupos de datos o varias variables en forma simultáneas, a partir de funciones discriminantes que son combinaciones lineales de las variables independientes que mejor discriminan o separan el grupo [53]. La Tabla ANOVA permite contrastar la hipótesis de igualdad de medias, que corresponde a la varianza entre los grupos en cada variable independiente, y también incluye el Lambda de Wilks univariable (Tabla 3), la cual equivale a la desviación a la media dentro de cada grupo; si su valor es pequeño, la variable discrimina mejor. Para esta gráfica es importante que el valor significante (sig) sea menor a 0,05 para ser aceptada; en caso contrario, no tiene sentido usar esa variable, ya que este valor califica la confiabilidad de los datos evaluados. Los resultados indican que las variables se podrían utilizar para el análisis, sin embargo, el Landa de Wilks permite seleccionar entre las variables pendiente e inclinación de la ladera, de acuerdo con su capacidad de discriminación, que está asociada a valores pequeños. La variable inclinación de la ladera tiene un valor Landa de Wilks de 0,915 , por lo que es seleccionada para los modelos.

Finalmente, el análisis de Componentes Principales para los componentes 1, 2 y 3 , representan cerca del $40 \%$ de la varianza del modelo (Fig. 4). Señala que las variables como WI y DI aportan en gran medida al componente principal $1, \mathrm{y}$ variables como TPI al componente principal 2, lo cual indica que favorecen la separación de datos. Evaluando los diferentes métodos de análisis, se evidencia un buen comportamiento de todas las variables en forma general, excepto en las variables inclinación de la ladera y pendiente, las cuales muestran una alta relación en todas las correlaciones; adicionalmente, en el análisis de Componentes Principales, se evidencia que la inclinación de la ladera aporta en gran medida al componente principal 1, que representa el $21 \%$ de la varianza total, por lo tanto se opta por utilizar esta variable y desestimar la pendiente. 
Métodos estadísticos para la evaluación de la susceptibilidad por movimientos en masa

Tabla 2. Análisis de Varianza (ANOVA). Fuente: autores.

\begin{tabular}{|c|c|c|c|c|c|c|c|c|c|c|c|}
\hline & & $\begin{array}{l}\text { Suma de } \\
\text { cuadrados }\end{array}$ & $\begin{array}{c}\text { Media } \\
\text { cuadrática }\end{array}$ & $\mathbf{F}$ & $\mathbf{S}$ & & & $\begin{array}{r}\text { Suma de } \\
\text { cuadrados }\end{array}$ & $\begin{array}{c}\text { Media } \\
\text { cuadrática }\end{array}$ & $\mathbf{a}$ & $\mathbf{S}$ \\
\hline \multirow{3}{*}{$\begin{array}{l}\text { D } \\
\text { V }\end{array}$} & E.G & 50266473 & 50266473 & 1886 & 0 & & E.G & 0 & 0 & 5250 & 0 \\
\hline & D.G & 179094150998 & 26648 & & & $\mathbf{R}$ & D.G & 35 & 0 & & \\
\hline & Total & 17959682471 & & & & & Total & 36 & & & \\
\hline \multirow{3}{*}{$\mathbf{A}$} & E.G & 8792284 & 8792284 & 857 & 0 & & E.G & 5731735 & 5731735 & 43741 & 0 \\
\hline & D.G & 6897839577 & 10264 & & & $\mathbf{S}$ & D.G & 88066219 & 131 & & \\
\hline & Total & 6906631861 & & & & & Total & 93797954 & & & \\
\hline \multirow{3}{*}{$\begin{array}{l}\mathbf{C} \\
\mathbf{P}\end{array}$} & E.G & & 1021777 & $\begin{array}{c}4478 \\
2\end{array}$ & 0 & & E.G & 153651 & 153651 & 0 & 0 \\
\hline & D.G & 15334366 & 23 & & & $\mathbf{P}$ & D.G & 1199516 & 2 & & \\
\hline & Total & 16356143 & & & & & Total & 1353167 & & & \\
\hline \multirow{3}{*}{$\begin{array}{l}\mathbf{C} \\
\mathbf{T}\end{array}$} & E.G & 23172 & 23172 & 2160 & 0 & $\mathbf{W}$ & E.G & 257630 & 257630 & & 0 \\
\hline & D.G & 7210080 & 11 & & & & D.G & 3944866 & 6 & & \\
\hline & Total & 7233252 & & & & & Total & 4202496 & & & \\
\hline \multirow{3}{*}{$\begin{array}{l}\text { D } \\
\text { F }\end{array}$} & E.G & 928481465 & 928481465 & 833 & 0 & $C$ & E.G & 0 & 0 & 0 & 0 \\
\hline & & $\begin{array}{c}749098770877 \\
0\end{array}$ & 1114621 & & & O & D.G & 1681632 & 3 & & \\
\hline & Total & 750027252334 & & & & B & Total & 1681633 & & & \\
\hline \multirow{3}{*}{$\begin{array}{l}\mathbf{G} \\
\mathbf{E} \\
\mathbf{O}\end{array}$} & E.G & 6217 & 6217 & 520 & 0 & & E.G & 15197679 & 15197679 & 9149 & 0 \\
\hline & D.G & 8039138 & 12 & & & $\begin{array}{l}\mathbf{D} \\
\mathbf{D}\end{array}$ & D.G & $\begin{array}{c}111643690 \\
2\end{array}$ & 1661 & & \\
\hline & Total & 8045355 & & & & $\mathbf{S}$ & Total & $\begin{array}{c}113163458 \\
2\end{array}$ & & & \\
\hline \multirow{3}{*}{$\begin{array}{l}\mathbf{R} \\
\mathbf{R}\end{array}$} & E.G & 1670577 & 1970577 & 282 & 0 & I & E.G & 15998207 & 15998207 & 56735 & 0 \\
\hline & D.G & 3975158457 & 5915 & & & $\mathbf{I}$ & D.G & 189509483 & 208 & & \\
\hline & Total & 3976829033 & & & & & Total & 205507690 & & & \\
\hline
\end{tabular}

*S = Sig E.G: Entre Grupos y D.G: Dentro de Grupos

Tabla 3. Resultados de Lambda de Wilks para cada variable. Fuente: autores.

\begin{tabular}{lcc}
\hline Mapas temáticos & Lambda & Sig. \\
\hline Distancia a vías & 0,886 & 0,000 \\
Aspecto & 0,834 & 0,000 \\
Curvatura perfil & 0,816 & 0,000 \\
Curvatura tangente & 0,804 & 0,000 \\
Distancia a Fallas & 0,800 & 0,000 \\
Geología & 0,797 & 0,000 \\
Relieve relativo & 0,795 & 0,000 \\
Rugosidad & 0,793 & 0,000 \\
Pendiente & 0,792 & 0,000 \\
Índice de posición topográfico & 0,791 & 0,000 \\
Índice de humedad topográfica & 0,790 & 0,000 \\
Cobertura & 0,789 & 0,000 \\
DDS & 0,789 & 0,000 \\
Inclinación de la ladera & 0,789 & 0,000 \\
\hline
\end{tabular}

[50] TecnoLógicas, ISSN-p 0123-7799 / ISSN-e 2256-5337, Vol. 22, No. 46, sep-dic de 2019, pp. 39-60 
En total se emplean las trece (13) variables restantes (inclinación de la ladera, coberturas, geología, relieve relativo, curvatura tangente, curvatura perfil, distancia al drenaje siguiendo la pendiente, rugosidad, distancia a fallas, distancia a vías, índice de posición topográfica, índice de humedad topográfica y aspecto), para correr el modelo.

La Fig. 5 y Fig. 6 presentan las variables categóricas y variables continuas, respectivamente, que se incorporan en el modelo de susceptibilidad.

\subsection{Modelo de susceptibilidad}

La Tabla 4 presenta los valores obtenidos para cada factor y clase, para
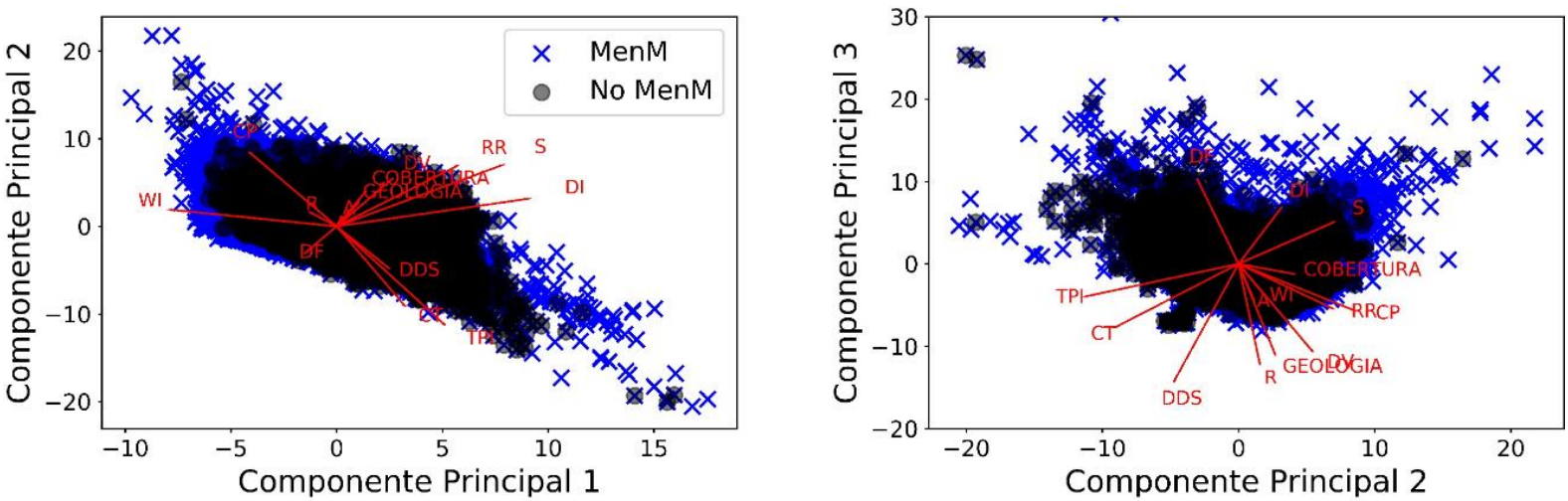

Fig. 4. Resultado del análisis de componentes principales. Fuente: autores.
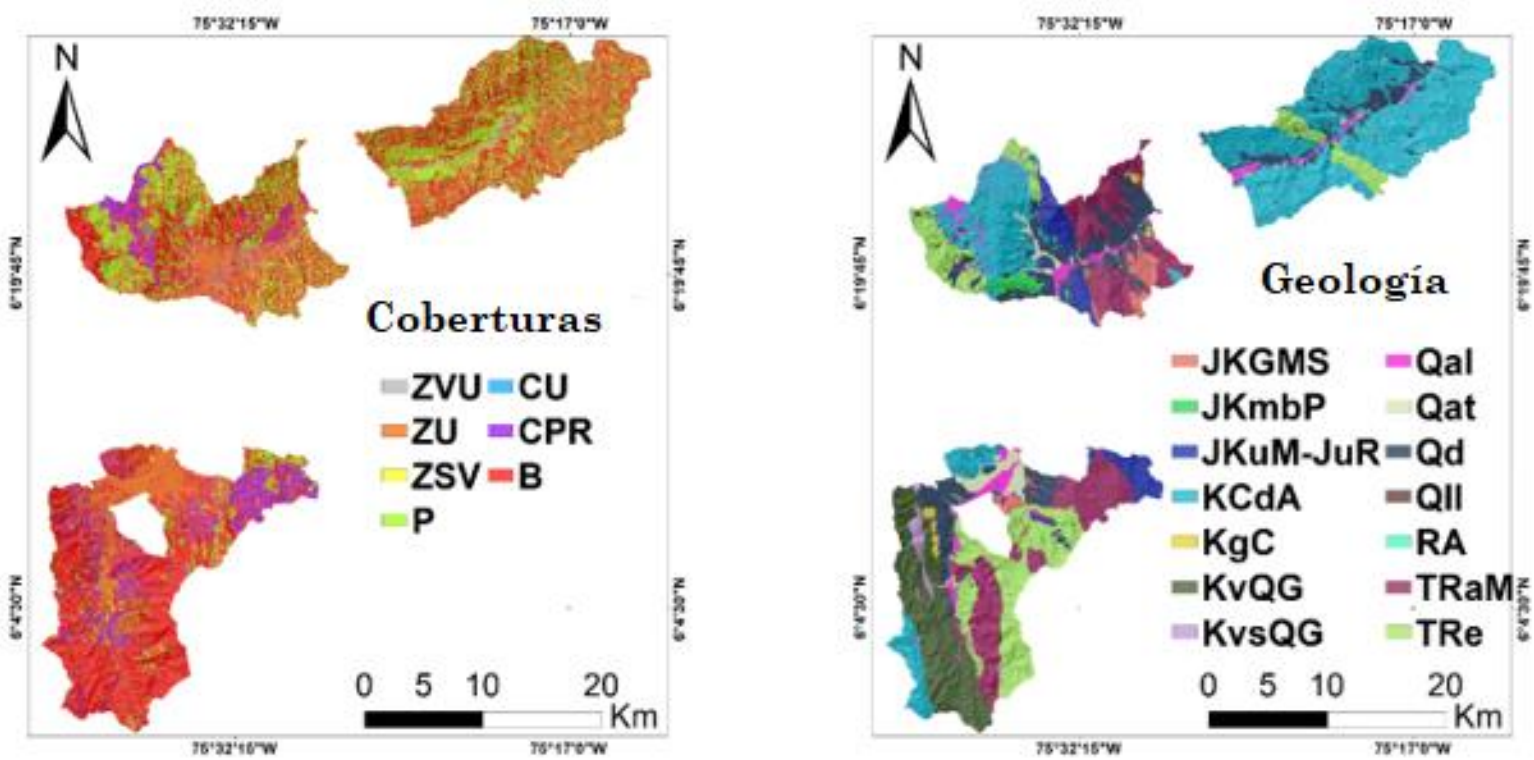

Fig. 5. Variables categóricas utilizadas en el modelo de susceptibilidad. Fuente: autores. 
Métodos estadísticos para la evaluación de la susceptibilidad por movimientos en masa
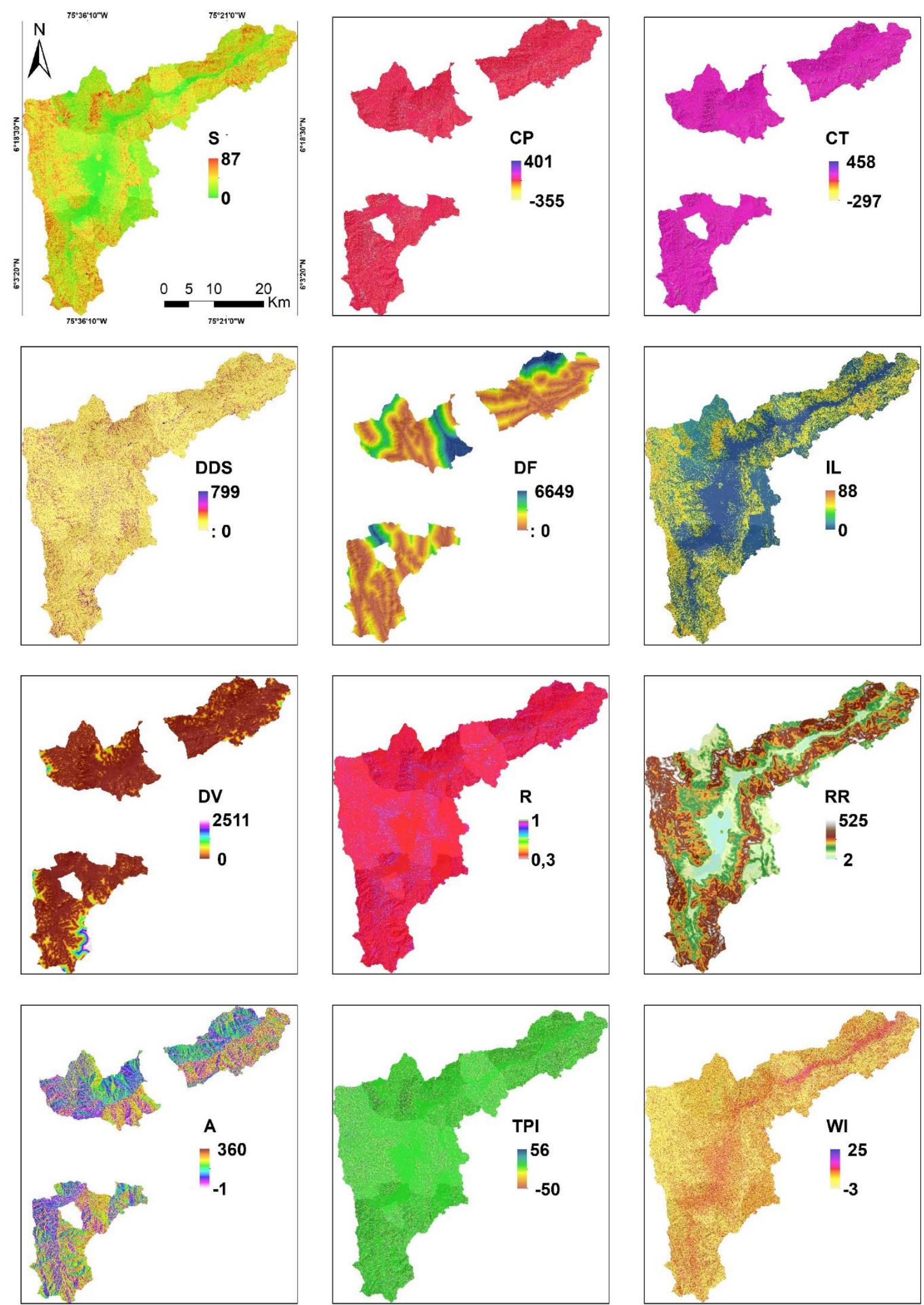

Fig. 6. Variables cuantitativas utilizadas en el modelo de susceptibilidad. Fuente: autores.

[52] TecnoLógicas, ISSN-p 0123-7799 / ISSN-e 2256-5337, Vol. 22, No. 46, sep-dic de 2019, pp. 39-60 
Métodos estadísticos para la evaluación de la susceptibilidad por movimientos en masa

Tabla 4. Factor calculado por Peso de la Evidencia y Regresión Logística para las categorías de los mapas temáticos del Valle de Aburrá. Fuente: autores.

\begin{tabular}{|c|c|c|c|c|c|c|c|c|c|c|c|}
\hline $\begin{array}{l}\text { Va } \\
\text { ria } \\
\text { ble }\end{array}$ & Rango & $\begin{array}{c}\text { WOE } \\
\text { (C) }\end{array}$ & RL & $\begin{array}{l}\text { Va } \\
\text { ria } \\
\text { ble }\end{array}$ & Rango & $\begin{array}{c}\text { WOE } \\
\text { (C) }\end{array}$ & RL & $\begin{array}{l}\text { Va } \\
\text { ria } \\
\text { ble }\end{array}$ & Rango & $\begin{array}{c}\text { WOE } \\
\text { (C) }\end{array}$ & RL \\
\hline \multirow{14}{*}{ 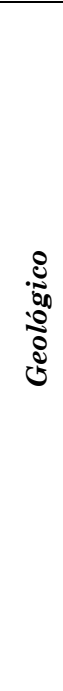 } & Anfibolitas & $-0,26$ & 0,16 & \multirow{8}{*}{ 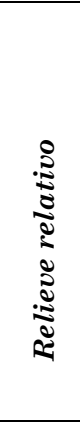 } & & & 0,003 & \multirow{7}{*}{$\begin{array}{c}0 \\
0 \\
0 \\
0 \\
0 \\
0 \\
0 \\
0 \\
0 \\
0 \\
0 \\
.00 \\
0\end{array}$} & & & 0,001 \\
\hline & Batolitos & 0,51 & 1,18 & & $0-40$ & $-2,12$ & & & $0-7$ & 0,19 & \\
\hline & $\begin{array}{l}\text { Depósito } \\
\text { Aluviotorrencial }\end{array}$ & $-1,16$ & 0,63 & & $40-60$ & $-0,48$ & & & 7 a 15 & 0,27 & \\
\hline & Depósito de vertiente & $-0,26$ & 0,538 & & $60-80$ & $-0,09$ & & & $15-30$ & 0,18 & \\
\hline & Depósitos aluviales & $-2,24$ & 0,97 & & $80-100$ & $-0,03$ & & & $30-45$ & 0,06 & \\
\hline & Dunita y peridotita & $-0,08$ & 0,648 & & $\begin{array}{l}100- \\
150\end{array}$ & 0,21 & & & $45-60$ & $-0,01$ & \\
\hline & Esquistos & $-0,08$ & 0,63 & & $\begin{array}{l}150- \\
200\end{array}$ & 0,24 & & & $>60$ & $-0,34$ & \\
\hline & Gabros & 0,26 & 0,323 & & $>200$ & $-0,12$ & & \multirow{10}{*}{$\begin{array}{l}0 \\
\stackrel{0}{0} \\
0 \\
\frac{0}{0} \\
\frac{\pi}{4}\end{array}$} & & & 0,001 \\
\hline & Llenos antrópicos & 0,09 & 0,659 & \multirow{8}{*}{ 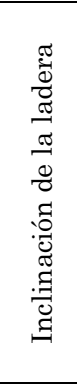 } & & & 0,051 & & -1 a 0 & $-2,42$ & \\
\hline & Metabasitas & 1 & 1,698 & & $<3$ & $-3,11$ & & & $0-45$ & 0,06 & \\
\hline & Neises y migmatitas & 0,26 & 0,454 & & 3 a 7 & $-2,19$ & & & $45-90$ & 0,08 & \\
\hline & Río Aburrá & $-6,63$ & 3,51 & & 7 a 15 & $-1,18$ & & & $90-135$ & 0,07 & \\
\hline & Volcánico & $-0,26$ & 0,472 & & $15-35$ & 0,7 & & & $135-180$ & 0,19 & \\
\hline & Vulcano sedimentario & $-0,4$ & 0 & & $35-50$ & 0,62 & & & $\begin{array}{l}180 \\
225\end{array}$ & 0,1 & \\
\hline \multirow{7}{*}{ 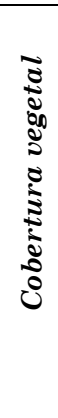 } & Zona urbana & $-0,37$ & 0,36 & & $50-70$ & 0,4 & & & $225-270$ & $-0,1$ & \\
\hline & $\begin{array}{l}\text { Zona desprovista de } \\
\text { vegetación }\end{array}$ & 0,39 & 0,384 & & $>70$ & $-0,31$ & & & $270-315$ & $-0,28$ & \\
\hline & Cuerpos de agua & $-3,31$ & 1,39 & \multirow{8}{*}{ 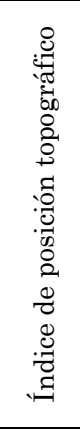 } & & & 0,556 & & $\begin{array}{l}315 \\
360\end{array}$ & $-0,14$ & \\
\hline & Pastos & 0,27 & 0,04 & & $<-10$ & $-5,48$ & & \multirow{7}{*}{ 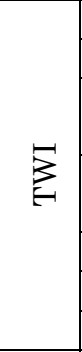 } & & & 0,065 \\
\hline & Zonas verdes urbanas & 0,03 & 0,13 & & $10 \mathrm{a}-1$ & $-1,29$ & & & $<2$ & 0,78 & \\
\hline & Bosques & $-0,18$ & 0,34 & & $\begin{array}{ll}-1 & a \\
0.5\end{array}$ & $-0,71$ & & & 2 a 6 & $-0,03$ & \\
\hline & $\begin{array}{l}\text { Cultivos y pastos } \\
\text { recreación rural }\end{array}$ & 0,14 & 0 & & $\begin{array}{ll}-0.5 & \mathrm{a} \\
0.5 & \end{array}$ & $-0,75$ & & & 6 a 8 & $-0,67$ & \\
\hline \multirow{6}{*}{ 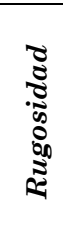 } & $<0.985$ & 0,46 & & & $0.5-1$ & 0,34 & & & $8 \mathrm{a} 10$ & $-1,02$ & \\
\hline & $0.985-0.99$ & 0,76 & & & 1 a 10 & 1,36 & & & 10 a 13 & $-1,35$ & \\
\hline & 0.99-0.995 & 0,8 & & & $>10$ & $-0,49$ & & & $13 \mathrm{a} 25$ & $-2,83$ & \\
\hline & $0.995-0.9975$ & 0,71 & & \multirow{8}{*}{ 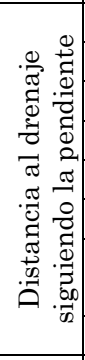 } & & & 0,001 & \multirow{6}{*}{ 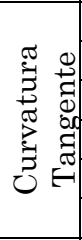 } & & & 0,108 \\
\hline & 0.9975-0.9985 & 0,51 & & & $0-15$ & $-1,31$ & & & $<-1,5$ & 0,26 & \\
\hline & $0.9985-1$ & $-0,96$ & & & $15-30$ & $-0,3$ & & & -0.1 & $-0,06$ & \\
\hline \multirow{6}{*}{ 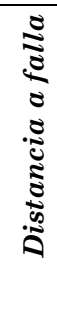 } & & & 0 & & $30-50$ & 0,38 & & & 0.1 & $-1,09$ & \\
\hline & $0-100$ & $-0,06$ & & & $50-70$ & 0,59 & & & 1.5 & $-0,35$ & \\
\hline & $100-300$ & 0,13 & & & $70-100$ & 0,33 & & & $>1,5$ & 0,41 & \\
\hline & $300-500$ & 0 & & & $\begin{array}{l}100 \\
180\end{array}$ & $-0,04$ & & \multirow{6}{*}{ 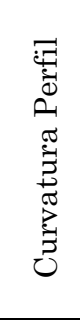 } & & & 0,02 \\
\hline & $500-1500$ & $-0,14$ & & & $>180$ & $-0,41$ & & & $<-1,5$ & 1,39 & \\
\hline & $>1500$ & 0,1 & & & & & & & -0.1 & $-0,27$ & \\
\hline & & & & & & & & & 0.1 & $-1,31$ & \\
\hline & & & & & & & & & 1.5 & $-0,82$ & \\
\hline & & & & & & & & & $>1,5$ & $-0,7$ & \\
\hline
\end{tabular}


Métodos estadísticos para la evaluación de la susceptibilidad por movimientos en masa

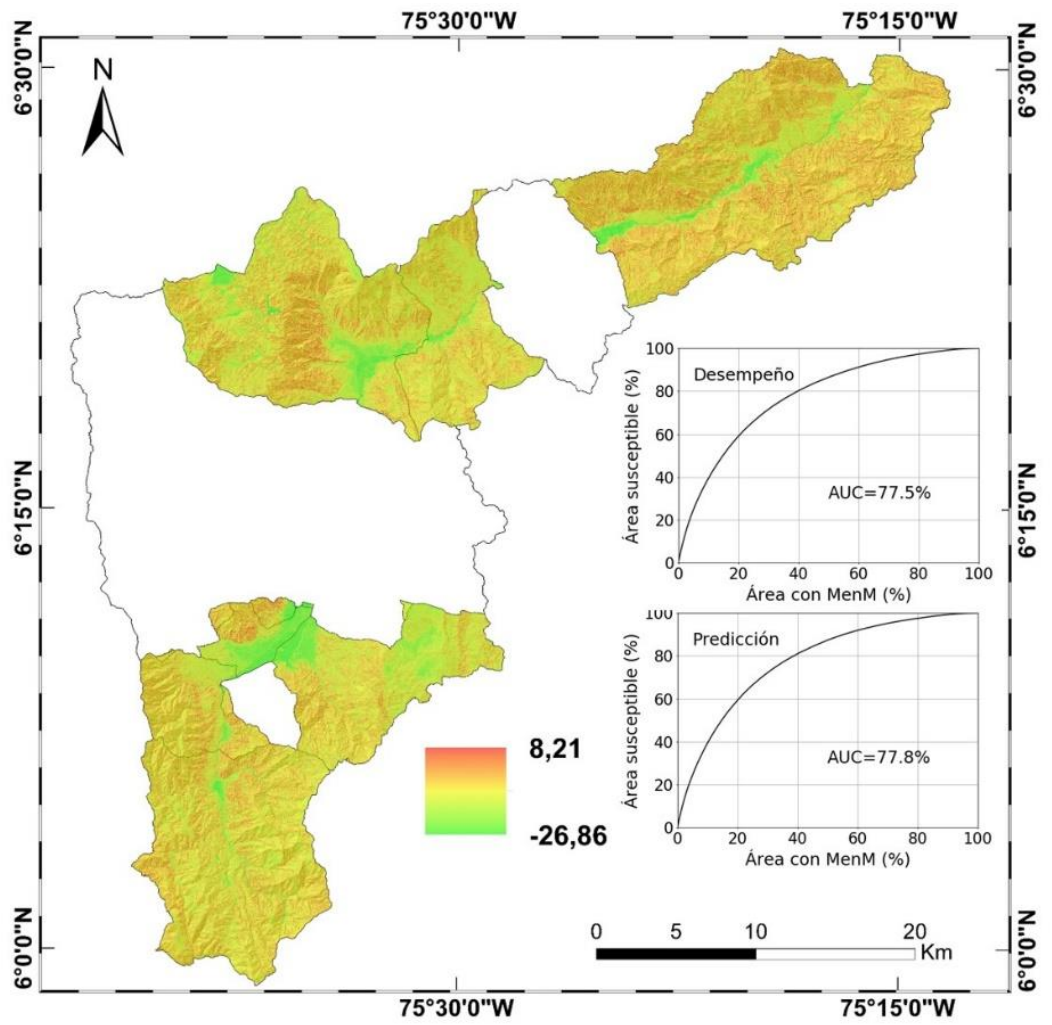

Fig. 7. Mapa de susceptibilidad por el método Peso de la Evidencia (WoE). Fuente: autores.

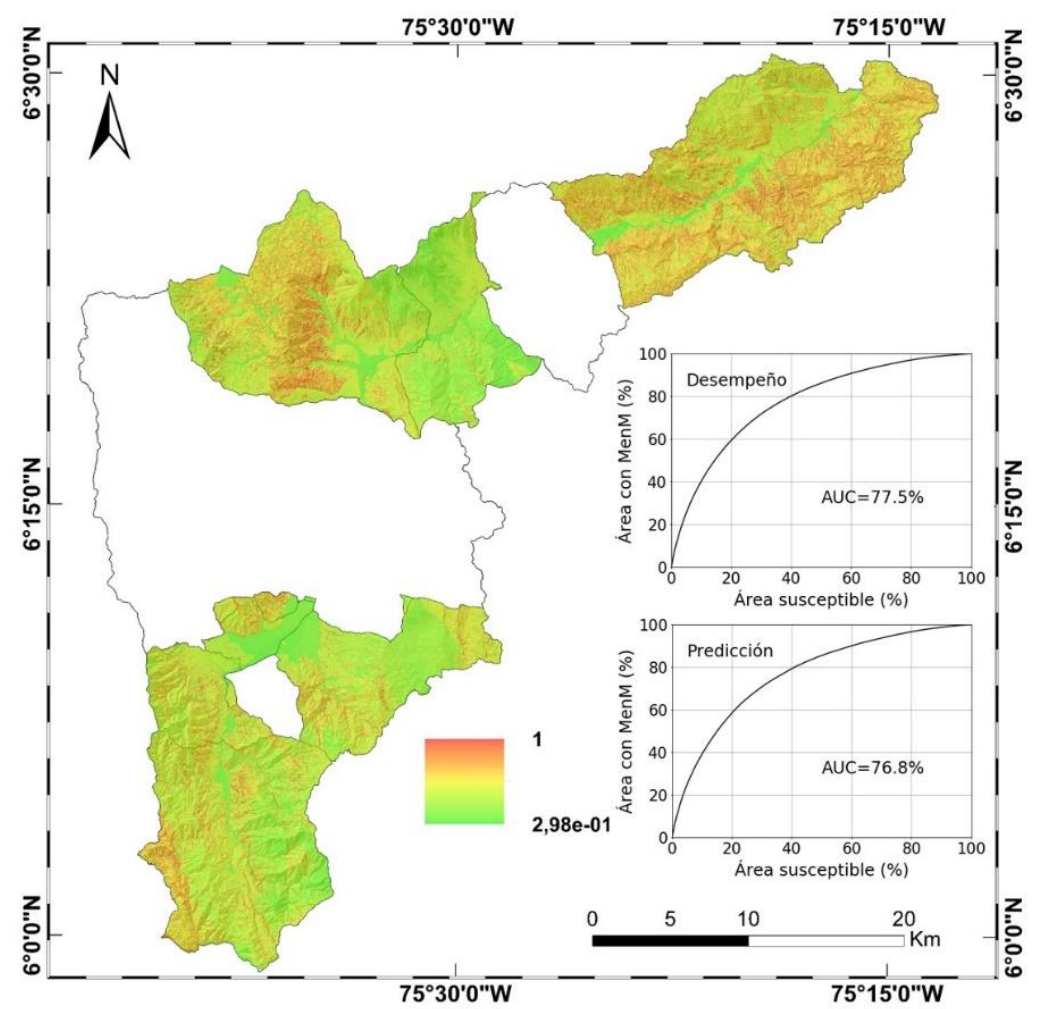

Fig. 8. Susceptibilidad por movimientos en masa para los municipios de estudio, obtenida mediante el modelo de regresión logística. Fuente: autores. 


\subsection{Selección y evaluación del modelo de susceptibilidad}

Los resultados señalan un desempeño y capacidad de predicción de ambos modelos, similar. El mapa obtenido con el método WoE presenta un valor ligeramente superior en la capacidad de predicción. Por lo tanto, al modelo WoE se le calculó la Matriz de Confusión, la cual es un método de validación, adicional a las curvas de éxito y predicción, que permite comparar las observaciones realizadas en el inventario de movimientos en masa con las predicciones del modelo. En el caso de estudio, esta se realizó con una muestra del $20 \%$ del total de las celdas de la zona. Para obtenerla, también se calculó el punto más cercano a la clasificación perfecta a partir de la curva ROC. Este punto correspondió a un valor de 0.38 , con el cual fue posible obtener los valores de la matriz de confusión y otros valores estadísticos que se derivan de esta, como radio de verdaderos positivos (TPR, por sus siglas en inglés), radio de falsaos positivos (FPR, por sus siglas en inglés), r (distancia a la clasificación perfecta), entre otros.

Los resultados se presentan en la Tabla 5 , en la cual la columna con nombre inventario positivo son las celdas con movimientos en masa identificados, e inventario negativo son las celdas sin movimientos en masa. Estos valores son comparados con los resultados del modelo WoE, con el fin de comparar el número de casos observados correcta e incorrectamente predichos por el modelo. El error del modelo está representado por los falsos positivos (FP), que corresponden al 28,3\%, y los falsos negativos (FN) que representan el 30,1\%. A su vez el acierto del modelo se puede estimar a través de los verdaderos positivos (TP) que corresponden al $69,9 \%$, y los verdaderos negativos (TN) que corresponden al $71.7 \%$ (Fig. 9). De tal forma, que es posible afirmar que el modelo de susceptibilidad es ligeramente más eficiente clasificando correctamente las laderas con ausencia de movimientos en masa.

La Tabla 6 presenta un resumen de los valores estadísticos obtenidos a partir de los métodos de validación del modelo de susceptibilidad. Los valores del área bajo la curva $(\mathrm{ABC})$ de éxito indican la habilidad del modelo para distinguir entre zonas susceptibles y no susceptibles a movimientos en masa. El hit rate, también conocido como sensitividad o TPR, representa la proporción de casos positivos predichos correctamente; a su vez, el radio de falsa alarma, o error negativo (FPR), es la relación entre los falsos positivos y el total de negativos. El valor de $r$ corresponde a la distancia a la clasificación perfecta (Fig. 9); y, finalmente, el radio odds utiliza todos los valores en la matriz de confusión, pues muestra la relación entre las observaciones correcta e incorrectamente clasificadas.

Tabla 5. Matriz de confusión. Fuente: autores.

\begin{tabular}{|c|c|c|c|c|c|c|c|c|}
\hline \multirow{2}{*}{ Predicción } & & \multicolumn{2}{|c|}{ Inventario Positivo } & \multirow{2}{*}{$\begin{array}{l}\text { Inventari } \\
\text { Celdas }\end{array}$} & \multirow{2}{*}{$\frac{\text { Negativo }}{\%}$} & & \multicolumn{2}{|c|}{ Total } \\
\hline & & Celdas & $\%$ & & & & Celdas & $\%$ \\
\hline WOE $>-0,38$ & $\mathrm{Si}$ & 29831848 & 70 & 29657 & 28,3 & Correcta & 29906826 & 70 \\
\hline WOE $<-0,38$ & No & 1281690 & 30 & 74981 & 71,7 & Incorrecta & 12846647 & 30 \\
\hline Total $\left(\sum\right)$ & & 42648838 & 100 & 104638 & 100 & & 42753473 & 100 \\
\hline
\end{tabular}

Tabla 6. Resultados de los diferentes métodos de validación utilizados, incluyendo ABC de las curvas de éxito y predicción, y valores estadísticos de la matriz de confusión. Fuente: autores.

\begin{tabular}{cccccc}
\hline $\begin{array}{c}\text { ABC Éxito } \\
\mathbf{( \% )}\end{array}$ & $\begin{array}{c}\text { ABC Predicción } \\
(\%)\end{array}$ & $\begin{array}{c}\text { Tasa Hir } \\
(\%)\end{array}$ & $\begin{array}{c}\text { Tasa de falsas } \\
\text { alarmas (\%) }\end{array}$ & Odds ratio & $\begin{array}{c}\text { Distancia a la } \\
\text { clasificación perfecta (r) }\end{array}$ \\
\hline 77,5 & 77,8 & 70 & 28 & 5,884 & 0,413 \\
\hline
\end{tabular}



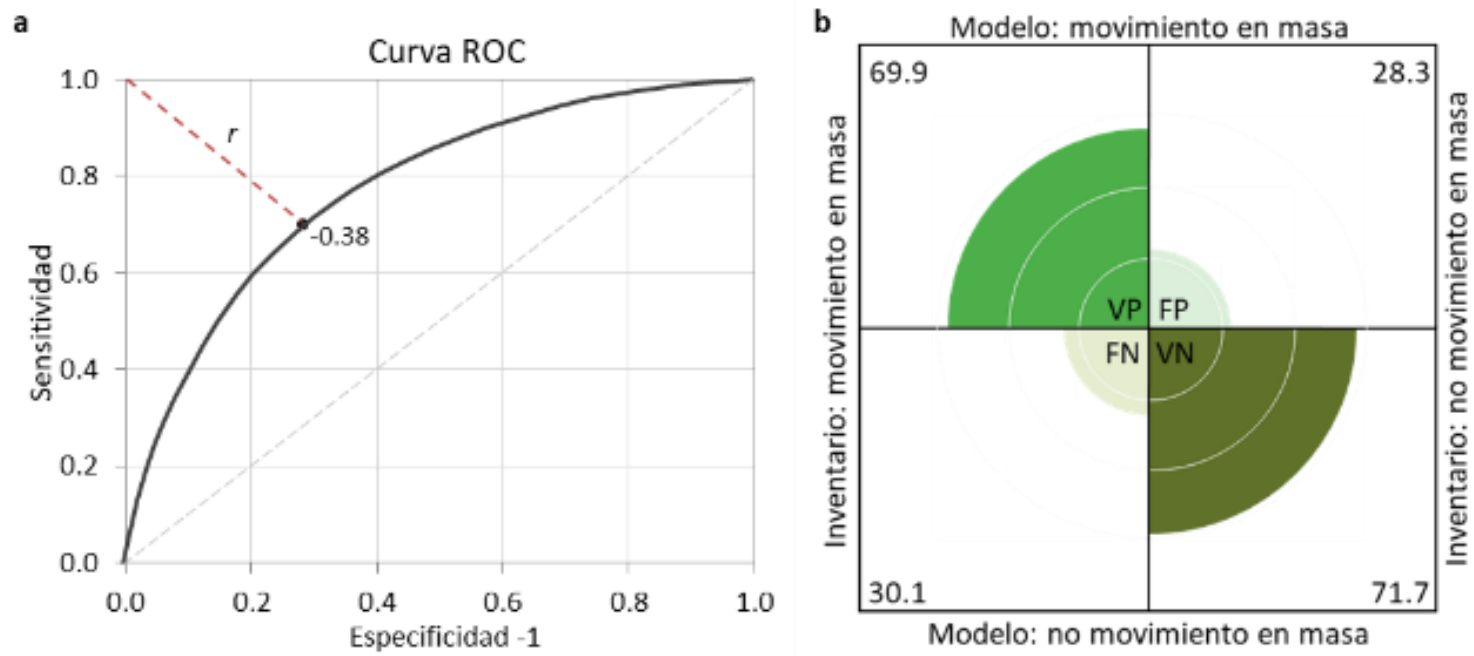

Fig. 9. Representación gráfica del punto más cercano a la clasificación perfecta (a) y de la matriz de confusión (b. Fuente: autores).

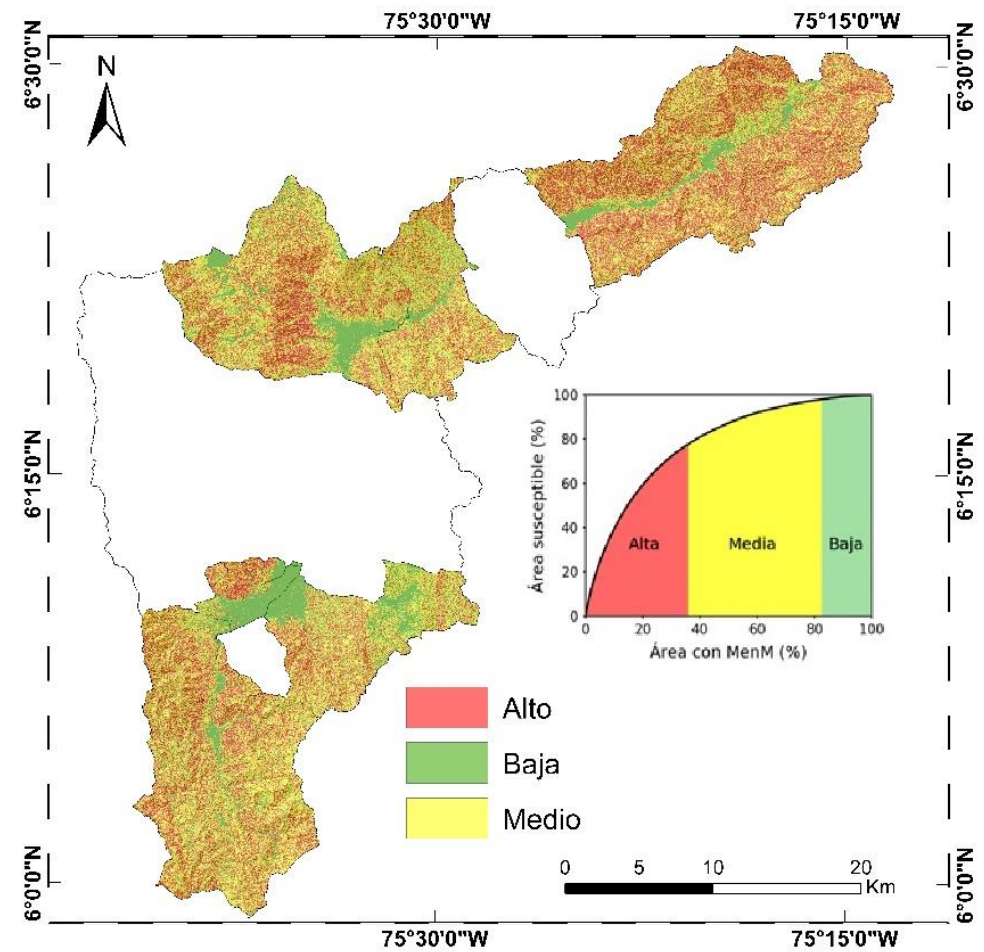

Fig. 10. Mapa de susceptibilidad por movimientos en masa para el valle de Aburrá. Fuente: autores.

La Fig. 10 presenta el mapa final de susceptibilidad por movimientos en masa en el valle de Aburrá. Este mapa es clasificado en tres categorías de acuerdo con la curva de éxito obtenida, donde se incluye en la categoría alta el $75 \%$ de los movimientos en masa, en la categoría media el $23 \%$, y en la categoría baja el 2 $\%$. Las zonas de susceptibilidad alta ocupa $227,8 \mathrm{~km}^{2}$ de los municipios de estudio, lo que representa el $33,3 \%$ del territorio y tiene una densidad de movimientos en 
masa de 66,1 MenM/km². La susceptibilidad media ocupa $342,9 \mathrm{~km}^{2}$ que representa 50,1 \% del área de interés, y tiene una densidad de movimientos en masa de $14,13 \quad \mathrm{MenM} / \mathrm{km}^{2}$. La susceptibilidad baja ocupa $113,28 \mathrm{~km}^{2}$ que representan el $16,56 \%$ de la zona de estudio, y tiene una densidad de movimientos en masa de $4,4 \mathrm{MenM} / \mathrm{km}^{2}$.

La Tabla 7 presenta para cada categoría de susceptibilidad el área total que ocupa dentro del área de estudio, el porcentaje y densidad de movimientos en masa que tiene asociados.

Tabla 7. Consolidado general de la susceptibilidad. Fuente: autores.

\begin{tabular}{lccc}
\hline Susceptibilidad & Alta & Media & Baja \\
\hline Probabilidad espacial & 2,2 & 0,5 & 0,1 \\
Área $\left(\mathrm{km}^{2}\right)$ & 227,77 & 342,88 & 113,28 \\
Área $(\%)$ & 33,3 & 50,13 & 16,56 \\
Área de M en M $\left(\mathrm{Km}^{2}\right)$ & 5,16 & 1,87 & 0,22 \\
Área de M en M (\%) & 71,13 & 25,84 & 3,03 \\
Densidad de M en M & 66,12 & 14,13 & 4,38 \\
$($ MenM/Km & \\
\hline
\end{tabular}

\section{CONCLUSIONES}

En el presente trabajo se presentaron los dos métodos estadísticos más ampliamente implementados para la evaluación y zonificación de la susceptibilidad por movimientos en masa. Asimismo, se elaboró un inventario a partir de registro histórico y fotointerpretación del área de estudio, lo que permitió tener un amplio y detallado conocimiento de los eventos pasados en el área de estudio, aportando 20,437 registros debidamente georeferenciados, que permiten mejorar el conocimiento actual de la morfodinámico actual e histórica del valle de Aburrá.

La selección de variables para la construcción del modelo estadístico se realizó mediante técnicas objetivas y cuantitativas, que permiten seleccionar las variables con mayor capacidad de predicción, y mejorar la eficiencia y eficacia del modelo, lo que señala la importancia de dichas técnicas para la construcción de un modelo de susceptibilidad adecuado. Ambos modelos estadísticos implementados en el área de estudio presentaron un desempeño similar, alrededor del $77 \%$, con un ligero aumento en la capacidad de predicción del modelo estadístico bivariado, WoE, confirmando que dicho método, propuesto por el SGC para los estudios básicos de amenaza para el ordenamiento territorial, representan una herramienta adecuada en la evaluación de la amenaza.

Finalmente, se resalta que el método presentado aporta un procedimiento objetivo y cuantitativo para la zonificación de la amenaza por movimientos en masa, que permite seleccionar el modelo más adecuado con criterios cuantitativos.

\section{REFERENCIAS}

[1] E. E. Brabb, "Innovative approaches to landslide hazard and risk mapping" conference paper in Proceedings of IVth International Conference and Field Workshop in Landslides, vol. 1, pp. 17-22, Tokyo, 1985.

https://pubs.er.usgs.gov/publication/7019752 9

[2] R. Soeters, R and C. J.V. Westen, Slope instability recognition, analysis and zonation, In: Turner, A.K., Schuster, R.L., Eds., Landslides: Investigation and Mitigation: TRB Sp. Washington D.C.: National Academy Press, 1996. https://research.utwente.nl/en/publications/sl ope-instability-recognition-analysis-andzonation

[3] P. Aleotti and R. Chowdhury, "Landslide hazard assessment: summary review and new perspectives," Bull. Eng. Geol. Environ., vol. 58, no. 1, pp. 21-44, Aug. 1999. https://doi.org/10.1007/s100640050066

[4] R. Fell, J. Corominas, C. Bonnard, L. Cascini, E. Leroi, and W. Z. Savage, "Guidelines for landslide susceptibility, hazard and risk zoning for land use planning," Eng. Geol., vol. 102, no. 3-4, pp. 85-98, Dec. 2008.

https://doi.org/10.1016/j.enggeo.2008.03.022

[5] C. J. van Westen, T. W. J. van Asch, and R. 
Soeters, "Landslide hazard and risk zonation-why is it still so difficult," Bull. Eng. Geol. Environ., vol. 65, no. 2, pp. 167184, May 2006.

https://doi.org/10.1007/s10064-005-0023-0

[6] F. Guzzetti, A. Carrara, M. Cardinali, and P. Reichenbach, "Landslide hazard evaluation: a review of current techniques and their application in a multi-scale study, Central Italy," Geomorphology, vol. 31, no. 1-4, pp. 181-216, Dec. 1999.

https://doi.org/10.1016/S0169.

555X(99)00078-1

[7] E. F. García-Aristizábal, E. V. Aristizabal Giraldo, R. J. Marín Sánchez, and J. C. Guzman Martinez, "Implementación del modelo TRIGRS con análisis de confiabilidad para la evaluación de la amenaza a movimientos en masa superficiales detonados por lluvia," TecnoLógicas, vol. 22, no. 44, pp. 111-129, Jan. 2019.

https://doi.org/10.22430/22565337.1037

[8] T. W. J. van Asch, J.-P. Malet, L. P. H. van Beek, and D. Amitrano, "Techniques, issues and advances in numerical modelling of landslide hazard," Bull. la Soc. Geol. Fr., vol. 178, no. 2, pp. 65-88, Mar. 2007. https://doi.org/10.2113/gssgfbull.178.2.65

[9] M. Casadei, W. E. Dietrich, and N. L. Miller, "Testing a model for predicting the timing and location of shallow landslide initiation in soil-mantled landscapes," Earth Surf. Process. Landforms, vol. 28, no. 9, pp. 925950, Aug. 2003.

https://doi.org/10.1002/esp.470

[10] S. Zhang, L. Zhao, R. Delgado-Tellez, and H. Bao, "A physics-based probabilistic forecasting model for rainfall-induced shallow landslides at regional scale," Nat. Hazards Earth Syst. Sci., vol. 18, no. 3, pp. 969-982, Mar. 2018.

https://doi.org/10.5194/nhess-18-969-2018

[11] C. Chung, "Using likelihood ratio functions for modeling the conditional probability of occurrence of future landslides for risk assessment," Comput. Geosci., vol. 32, no. 8, pp. 1052-1068, Oct. 2006.

https://doi.org/10.1016/j.cageo.2006.02.003

[12] T. Chen, R. Niu, and X. Jia, "A comparison of information value and logistic regression models in landslide susceptibility mapping by using GIS," Environ. Earth Sci., vol. 75, no. $10, \quad$ p. 867 , May 2016. https://doi.org/10.1007/s12665-016-5317-y

[13] C. J. Van Westen, "Application of geographic information systems to landslide hazard zonation," ITC, Ensched 1993. https://research.utwente.nl/en/publications/a pplication-of-geographic-informationsystems-to-landslide-hazard
[14] C.-J. F. Chung and A. G. Fabbri, "The representation of geoscience information for data integration," Nonrenewable Resour., vol. 2, no. 2, pp. 122-139, Jun. 1993. https://doi.org/10.1007/BF02272809

[15] P. V. Gorsevski, P. Gessler, and R. B. Foltz, "Spatial Prediction of Landslide Hazard Using Discriminant Analysis and GIS.," in GIS in the Rockies 2000 Conference and Workshop Applications for the 21st Century, 2000.

[16] L. Ayalew and H. Yamagishi, "The application of GIS-based logistic regression for landslide susceptibility mapping in the Kakuda-Yahiko Mountains, Central Japan," Geomorphology, vol. 65, no. 1-2, pp. 15-31, Feb. 2005.

https://doi.org/10.1016/j.geomorph.2004.06.01 0

[17] Organización de Estados Americanos (OEA) "Chapter 10: Landslide hazard assessment. In: Primer on natural hazard management in integrated regional development planning. Dept. of Regional Development and Environment, Executive Secretariat for Economic and Social Affairs (DRDE), General Secretariat, Washington, DC.," Washington, 1991.

[18] A. Clerici, S. Perego, C. Tellini, and P. Vescovi, "A procedure for landslide susceptibility zonation by the conditional analysis method," Geomorphology, vol. 48, no. 4, pp. 349-364, Dec. 2002.

https://doi.org/10.1016/S0169555X(02)00079-X

[19] A. Brenning, "Spatial prediction models for landslide hazards: review, comparison and evaluation," Nat. Hazards Earth Syst. Sci., vol. 5, no. 6, pp. 853-862, Nov. 2005. https://doi.org/10.5194/nhess-5-853-2005

[20] S. Lee and B. Pradhan, "Landslide hazard mapping at Selangor, Malaysia using frequency ratio and logistic regression models," Landslides, vol. 4, no. 1, pp. 33-41, Mar. 2007. https://doi.org/10.1007/s10346006-0047-y

[21] I. Cantarino, M. A. Carrion, F. Goerlich, and V. Martinez Ibañez, "A ROC analysis-based classification method for landslide susceptibility maps," Landslides, vol. 16, no. 2, pp. 265-282, Feb. 2018. https://doi.org/10.1007/s10346-018-1063-4

[22] D. Cheng, Y. Cui, F. Su, Y. Jia, and C. E. Choi, "The characteristics of the Mocoa compound disaster event, Colombia," Landslides, vol. 15, no. 6, pp. 1223-1232, Jun. 2018. https://doi.org/10.1007/s10346018-0969-1

[23] R. J. Marín, J. C. Guzmán-Martínez, H. E. Martínez Carvajal, E. F. García-Aristizábal, 
J. D. Cadavid-Arango, and P. AgudeloVallejo, "Evaluación del riesgo de deslizamientos superficiales para proyectos de infraestructura: caso de análisis en vereda El Cabuyal,”Ing. y Cienc., vol. 14, no. 27, pp. 153-177, Jun. 2018.

https://doi.org/10.17230/ingciencia.14.27.7

$[24]$

E. Aristizábal and J. Gómez, "Inventario de emergencias y desastres en el Valle de Aburrá originados por fenómenos naturales y antrópicos en el período 1880-2007," Rev. Gestión y Ambient., vol. 10, N, pp. 17-30, https://doi.org/10.15446/ga

[25] É. Aristizábal, "Características, dinámica y causas del movimiento en masa del barrio el socorro (31 de mayo de 2008) en Medellín," Rev. EIA, vol. 10, pp. 19--29., jun. 2008. http://www.scielo.org.co/scielo.php?script=sci arttext\&pid=S1794-12372008000200003

[26] Integral S.A, U EAFIT, Universidad Nacional de Colombia, Intenisa. S.A, and Solingral. S.A, "Microzonificación sísmica detallada de los municipios de Barbosa, Girardota, Copacabana, Sabaneta, La Estrella, Caldas y Envigado.," 2006. https://repositorio.gestiondelriesgo.gov.co/ha ndle/20.500.11762/19862

[27] G. Rodríguez, H. González, G. Zapata, U. Cossio, and A. M. C. Martínez, "Geología de la plancha 147 Medellín Oriental Escala 1:50.000 Versión 2016," Medellín, 2016. https://www.researchgate.net/publication/32 0740761_geologia_de_la_plancha_147_medel lin_oriental_escala_150000_version_2016

[28] F. Guzzetti, P. Reichenbach, F. Ardizzone, M. Cardinali, and M. Galli, "Estimating the quality of landslide susceptibility models," Geomorphology, vol. 81, no. 1-2, pp. 166184, Nov. 2006.

https://doi.org/10.1016/j.geomorph.2006.04.00 7

[29] Servicio Geológico Colombiano - SGC, "Guía Metodológica para estudios de Amenaza, Vulnerabilidad y Riesgo por movimientos en masa.," 2015.

https://www2.sgc.gov.co/Noticias/boletinesDo cumentos/1642_Guia-Metodologica-27-072016-SinGuias.pdf

[30] G. Herrera et al., "Landslide databases in the Geological Surveys of Europe," Landslides, vol. 15, no. 2, pp. 359-379, Feb. 2018. https://doi.org/10.1007/s10346-0170902-z

[31] Corporación OSSO, "Que es DesInventar," Sept, 2017. https://www.desinventar.org/es/

[32] Servicio Geológico Colombiano SGC, "SIMMA.," 2017. http://simma.sgc.gov.co/\#/

[33] J. Corominas et al., "Recommendations for the quantitative analysis of landslide risk," Bull. Eng. Geol. Environ., no 2. vol. 73, pp.
209-- 263., Nov. 2013.

https://doi.org/10.1007/s10064-013-0538-8

[34] T. Glade and M. J. Crozier, "A Review of Scale Dependency in Landslide Hazard and Risk Analysis," in Landslide Hazard and Risk, vol. 75, Chichester, West Sussex, England: John Wiley \& Sons, Ltd, 2012. pp. $75-138$.

https://doi.org/10.1002/9780470012659.ch3

[35] C.-T. Lee, C.-C. Huang, J.-F. Lee, K.-L. Pan, M.-L. Lin, and J.-J. Dong, "Statistical approach to storm event-induced landslides susceptibility," Nat. Hazards Earth Syst. Sci., vol. 8, no. 4, pp. 941-960, Aug. 2008. https://doi.org/10.5194/nhess-8-941-2008

[36] M. L. Süzen and V. Doyuran, "Data driven bivariate landslide susceptibility assessment using geographical information systems: a method and application to Asarsuyu catchment, Turkey," Eng. Geol., vol. 71, no. 3-4, pp. 303-321, Feb. 2004.

https://doi.org/10.1016/S0013-7952(03)001431

[37] P. Atkinson, H. Jiskoot, R. Massari, and T. Murray, "Generalized linear modelling in geomorphology," Earth Surf. Process. Landforms, vol. 23, no. 13, pp. 1185-1195, Dec. 1998.

https://doi.org/10.1002/(SICI)1096-

9837(199812)23:13<1185::AID-

ESP928>3.0.CO;2-W

[38] B. A. Olshausen, "Bayesian probability theory." pp. 1-6, 2004. http://www.rctn.org/bruno/npb163/bayes.pdf

[39] E. R. Sujatha, P. Kumaravel, and G. V. Rajamanickam, "Assessing landslide susceptibility using Bayesian probabilitybased weight of evidence model," Bull. Eng. Geol. Environ., vol. 73, no. 1, pp. 147-161, Feb. 2014. https://doi.org/10.1007/s10064013-0537-9

[40] N. R. Regmi, J. R. Giardino, and J. D. Vitek, "Modeling susceptibility to landslides using the weight of evidence approach: Western Colorado, USA," Geomorphology, vol. 115, no. 1-2, pp. 172-187, Feb. 2010.

https://doi.org/10.1016/j.geomorph.2009.10.00 2

[41] C. J. van Westen, N. Rengers, and R. Soeters, "Use of Geomorphological Information in Indirect Landslide Susceptibility Assessment," Nat. Hazards, vol. 30, no. 3, pp. 399-419, Nov. 2003. https://doi.org/10.1023/B:NHAZ.0000007097. $42735.9 \mathrm{e}$

[42] H.-J. Oh and S. Lee, "Landslide susceptibility mapping on Panaon Island, Philippines using a geographic information system," Environ. Earth Sci., vol. 62, no. 5, pp. 935-951, Mar. 2011. 
https://doi.org/10.1007/s12665-010-0579-2

[43] R. K. Dahal and S. Hasegawa, "Representative rainfall thresholds for landslides in the Nepal Himalaya," Geomorphology, vol. 100, no. 3-4, pp. 429443, Aug. 2008.

https://doi.org/10.1016/j.geomorph.2008.01.01 4

[44] A. Ozdemir and T. Altural, "A comparative study of frequency ratio, weights of evidence and logistic regression methods for landslide susceptibility mapping: Sultan Mountains, SW Turkey," J. Asian Earth Sci., vol. 64, no. 5, pp. 180-197, Mar. 2013.

https://doi.org/10.1016/j.jseaes.2012.12.014

[45] Servicio Geológico Colombiano - SGC, "Guía Metodológica para Zonificación de Amenaza por Movimientos en Masa a escala 1:25.000.," Bogotá, 2017.

https://www2.sgc.gov.co/Archivos/GM(2017). pdf

[46] P. M. Atkinson and R. Massari, "Generalised linear modelling of susceptibility to landsliding in the central apennines, italy," Comput. Geosci., vol. 24, no. 4, pp. 373-385, May 1998. https://doi.org/10.1016/S00983004(97)00117-9

[47] Australian Geomechanics Society, "Australian Geomechanics," Australia. 2007. https://australiangeomechanics.org/

[48] M. T. Davis, P.A, Goodrich, "A proposed strategy for the validation of ground-water flow and solute transport models"., vol. 23, no. 9. Albuquerque, NM,Nuclear Energy Agency of the OECD (NEA), 1991.

[49] C.-J. F. Chung and A. G. Fabbri, "Validation of Spatial Prediction Models for Landslide Hazard Mapping," Nat. Hazards, vol. 30, no. 3, pp. 451-472, Nov. 2003. https://doi.org/10.1023/B:NHAZ.0000007172. $62651.2 \mathrm{~b}$

[50] T. Fawcett, "An introduction to ROC analysis," Pattern Recognit. Lett., vol. 27, no. 8, pp. 861-874, Jun. 2006. https://doi.org/10.1016/j.patrec.2005.10.010

[51] G. G. Chevalier, V. Medina, M. Hürlimann, and A. Bateman, "Debris-flow susceptibility analysis using fluvio-morphological parameters and data mining: application to the Central-Eastern Pyrenees," Nat. Hazards, vol. 67, no. 2, pp. 213-238, Jun. 2013. https://doi.org/10.1007/s11069-0130568-3

[52] D. J. Cruden, D. M., \& Varnes, "Landslides: investigation and mitigation. Chapter 3Landslide types and processes.," in Transportation research board special report, vol. 247, Transportation Research Board,washington 1996, pp. 36-75.

[53] Z. Jing, G. Wang, , S. Zhang, \& C. Qiu, "Building Tianjin driving cycle based on linear discriminant analysis".Transportation Research Part D: Transport and Environment, 53, 78-87, Jun. 2017. https://doi.org/10.1016/j.trd.2017.04.005 